\title{
UNDERSTANDING BOOMS AND BUSTS IN HOUSING MARKETS
}

\author{
Craig Burnside \\ Martin Eichenbaum \\ Sergio Rebelo \\ Working Paper 16734 \\ http://www.nber.org/papers/w16734 \\ NATIONAL BUREAU OF ECONOMIC RESEARCH \\ 1050 Massachusetts Avenue \\ Cambridge, MA 02138 \\ January 2011
}

We thank Eric Aldrich, Fernando Alvarez, Veronica Guerrieri, Peter Howitt, John Leahy, Martin Schneider, Rob Shimer, and Susan Woodward for their comments. The views expressed herein are those of the authors and do not necessarily reflect the views of the National Bureau of Economic Research.

(C) 2011 by Craig Burnside, Martin Eichenbaum, and Sergio Rebelo. All rights reserved. Short sections of text, not to exceed two paragraphs, may be quoted without explicit permission provided that full credit, including $(\mathcal{C}$ notice, is given to the source. 
Understanding Booms and Busts in Housing Markets

Craig Burnside, Martin Eichenbaum, and Sergio Rebelo

NBER Working Paper No. 16734

January 2011, Revised May 2011

JEL No. E32,R31

\section{ABSTRACT}

Some booms in housing prices are followed by busts. Others are not. In either case it is difficult to find observable fundamentals that are correlated with price movements. We develop a model consistent with these observations. Agents have heterogeneous expectations about long-run fundamentals but change their views because of "social dynamics." Agents meet randomly. Those with tighter priors are more likely to convert others to their beliefs. The model generates a "fad": the fraction of the population with a particular view rises and then falls. Depending on which agent is correct about fundamentals, these fads generate boom-busts or protracted booms.

Craig Burnside

Department of Economics

Duke University

213 Social Sciences Building

Durham, NC 27708-0097

and NBER

burnside@econ.duke.edu

Martin Eichenbaum

Department of Economics

Northwestern University

2003 Sheridan Road

Evanston, IL 60208

and NBER

eich@northwestern.edu
Sergio Rebelo

Northwestern University

Kellogg School of Management

Department of Finance

Leverone Hall

Evanston, IL 60208-2001

and NBER

s-rebelo@northwestern.edu 


\section{Introduction}

Some booms in housing prices are followed by busts. Others are not. In either case it is difficult to find observable fundamentals that are correlated with price movements. We develop a model that is consistent with these observations. Agents have heterogeneous expectations about long-run fundamentals but change their views because of "social dynamics". Agents meet randomly and those with tighter priors are more likely to convert other agents to their beliefs. The model generates a "fad" in the sense that the fraction of the population with a particular view rises and then falls. Depending on which agent is correct about fundamentals, these fads generate boom-busts or protracted booms.

Models in which agents have homogeneous expectations can generate large differences in house prices across steady states with different fundamentals such as borrowing constraints, income growth, demographics, transactions costs, and zoning (see e.g. Chu (2009)). However, it is difficult to generate protracted price movements in models with homogeneous expectations because expected changes in future fundamentals are quickly capitalized into prices. Booms and busts can be generated by assuming that agents first receive increasingly positive signals about future fundamentals and then increasingly negative signals. But the problem with this approach is that for many episodes it is difficult to find observable fundamentals that are correlated with house price movements. ${ }^{1}$

The model that we develop has three key features. First, there is uncertainty about the long-run fundamentals that drive house prices. We assume that in each period, there is a small probability that housing fundamentals will change permanently to a new value. This emphasis on long-run fundamentals is related to the literature on long-run risk (Bansal and Yaron (2004), and Hansen, Heaton, and Li (2008)).

Second, as in Harrison and Kreps (1976), Scheinkman and Xiong (2003), Acemoglu, Chernozhukov, and Yildiz (2007), and Geanakoplos (2010), agents in our economy have heterogenous beliefs about fundamentals. Some agents believe that housing fundamentals will improve while others don't. Agents can update their priors in a Bayesian fashion. But the data do not convey useful information about long-run fundamentals, so the priors of different agents remain constant over time. In other words, agents agree to disagree and this disagreement persists over time. One set of agents is correct in their views but there is no

\footnotetext{
${ }^{1}$ Glaeser and Gyourko (2006) argue that it is difficult to explain the large changes in housing prices over time with changes in incomes, amenities or interest rates.
} 
way to know ex-ante which set that is.

The third feature of the model is an element which we refer to as "social dynamics." Agents meet randomly with each other and some agents change their priors about long-run fundamentals as a result of these meetings. We use the entropy of an agent's probability distribution of future fundamentals to measure the uncertainty of his views. We assume that when agent $i$ meets agent $j$, the probability that agent $i$ adopts the prior of agent $j$ is decreasing (increasing) in the entropy of agent $i$ 's (agent $j$ 's) priors. Agents with tighter priors are more likely to convert other agents to their beliefs. Our model generates dynamics in the fraction of agents who hold different views that are similar to those generated by the infectious disease models proposed by Bernoulli (1766) and Kermack and McKendrick (1927). Taken together, the second and third features of the model generate non-trivial dynamics in the volume of transactions, with home sales and prices displaying a sharp positive correlation.

We consider two cases. In the first case the agents with the tightest priors are those who expect fundamentals to remain the same. In the second case, agents with the tightest priors are those who expect fundamentals to improve. Absent realization of uncertainty about long-run fundamentals, the model generates fads. In the first case there is a rise and fall in the number of people who believe that buying a house is a good investment. Here the model generates a protracted boom-bust cycle. In the second case there is a rise and fall in the number of people who believe that housing fundamentals will not change. Here, the model generates a protracted boom in housing prices that is not followed by a bust.

We use the model to compute the price path expected by different agents. These unconditional expected price paths take into account the probability of uncertainty being realized at different points in time. Regardless of which agent happens to be correct the model has the following implications. Agents who think that fundamentals will improve expect prices to rise and then level off. Agents who think fundamentals will not change expect prices to rise and then fall. An econometrician taking repeated samples from data generated by the model would see both boom-busts and booms that are not followed by busts. The boom-bust episodes occur in economies where agents who don't expect fundamentals to improve happen to be correct. The episodes in which booms are not followed by busts occur in economies where those agents who expect fundamentals to improve happen to be correct. Of course, in any given economy the econometrician could not predict ex-ante which type of episode he would see. That is because before uncertainty is realized, the data are not informative 
about which agent is correct.

The paper is organized as follows. In Section 2 we provide some empirical background about the nature of housing booms and busts. In Section 3 we describe our model of social dynamics and its implications for the behavior of housing prices. We do so in a frictionless model of the housing market. While useful for building intuition, the model is too stylized to account for various features of the data. For this reason we consider in Section 4 a simple matching model of the housing market and describe its transition dynamics. In Section 5 we incorporate social dynamics into the matching model and generate our main results. Section 6 concludes.

\section{Boom-bust cycles in housing prices: background evidence}

In this section we document some key empirical regularities about boom-bust cycles in housing prices using aggregate time-series data for different countries. Our evidence complements the already extensive literature that uses microeconomic data to analyze particular boombust episodes (see e.g. Mian and Sufi (2010) and Barlevy and Fisher (2010)).

An operational definition of a boom or a bust requires that we define turning points where upturns and downturns in housing prices begin. To avoid defining high-frequency movements in the data as upturns or downturns, we first smooth the data. Let $y_{t}$ denote the logarithm of an index of real housing prices. Also let $x_{t}$ denote the centered-moving average of $y_{t}, x_{t}=\sum_{j=-n}^{n} y_{t+j}$. We define an upturn as an interval of time in which $\Delta x_{t}>0$ for all $t$ and a downturn as an interval of time in which $\Delta x_{t}<0$. A turning point is the last time period within an upturn or downturn. A boom is an upturn for which $y_{T}-y_{T-L}>z$, and a bust is a downturn for which $y_{T}-y_{T-L}<-z$. Here $T$ is the date at which the boom or bust ended, $L$ is the length of the boom or bust and $z$ is a positive scalar. The results discussed below are generated assuming $n=5$ and $z=0.15$ but the findings are not sensitive to small changes in these parameters.

We implement our procedure using quarterly OECD data on real house prices for 18 countries over the period 1970 to $2009 .^{2}$ These indices have been normalized so they have an average value of 100 in all countries. Figure 1 displays the data. Three features of these data

\footnotetext{
${ }^{2}$ While our data spans the last four decades, boom-bust episodes in housing prices are a much older phenomenon. Ambrose, Eichholtz, Lindenthal (2010) document the existence of such episodes in Holland over a period of four centuries. Similarly, Eitrheim and Erlandsen (2004) provide analogous evidence for Norway over a period of two centuries.
} 
are worth noting. First, every country in our sample experienced housing price booms and busts. $^{3}$ The median sizes of booms and busts are 54 and 29 percent, respectively. Second, booms and busts occur over protracted periods of time. The median lengths of booms and busts in our sample are $6 \frac{1}{4}$ years and 5 years, respectively. Third, in many cases booms are followed by protracted busts. But not always: in 26 out of 49 boom episodes a boom is not followed by a bust. ${ }^{4}$ A successful theory should recognize this fact.

\section{Social dynamics in a frictionless model}

In this section we consider a simple frictionless model of the housing market. We use this set up to introduce our model of social dynamics and the implied movements in the fraction of agents with different beliefs about long-run fundamentals.

The model economy The economy is populated by a continuum of agents with measure one. All agents have linear utility and discount utility at rate $\beta$. Agents are either homeowners or renters. We assume that each agent can only own one house and that there is no short-selling. The first assumption is made for simplicity. The second assumption is motivated by the fact that in practice it is not possible to short sell houses. This characteristic of houses distinguishes it from other asset classes, such as stocks, which are easier to short sell.

For simplicity, we assume that there is a fixed stock of houses, $k<1$, in the economy. This assumption is motivated by the observation that large booms and busts occur in cities where increases in the supply of houses are limited by zoning laws, land scarcity, or infrastructure constraints. $^{5}$ There is a rental market with $1-k$ houses. These units are produced by competitive firms at a cost of $w$ per period, so the rental rate is constant and equal to $w$. The momentary utilities associated with owning and renting a house are $\varepsilon^{h}$ and $\varepsilon^{r}$, respectively. We assume that the utility of owning a home is higher than the net utility of renting $\left(\varepsilon^{h}>\varepsilon^{r}-w\right)$ so that home prices are positive.

We first consider the equilibrium of the economy when there is no uncertainty. Agents decide at time $t$ whether they will be renters or home owners at time $t+1$. The net utility

\footnotetext{
${ }^{3}$ Australia and Germany only experienced booms and a bust, respectively.

${ }^{4}$ This fraction is almost certainly affected by the fact that our sample ends in 2009 and so misses part of the ongoing declines in home prices.

${ }^{5}$ See, e.g. Glaeser, Gyourko, and Saks (2005), Quigley and Raphael (2005), Barlevy and Fisher (2010), and Saiz (2010).
} 
of being a renter at time $t+1$ is $\varepsilon^{r}-w$. If an agent buys a house at time $t$ he pays $P_{t}$. He lives in the home at time $t+1$ and receives a utility flow $\varepsilon^{h}$. He can then sell the house at the end of period $t+1$ for a price $P_{t+1}$. Since all agents are identical, in equilibrium they must be indifferent between buying and renting a house. So, housing prices must satisfy the following equation:

$$
-P_{t}+\beta\left(P_{t+1}+\varepsilon^{h}\right)=\beta\left(\varepsilon^{r}-w\right) .
$$

The stationary solution to this equation is: ${ }^{6}$

$$
P=\beta \frac{\varepsilon}{1-\beta}
$$

where $\varepsilon=\varepsilon^{h}-\left(\varepsilon^{r}-w\right)$.

We now consider an experiment that captures the effects of infrequent changes in the value of housing fundamentals. Examples include low-frequency changes in the growth rate of productivity which affects agents' wealth and changes in financial regulation or innovations which make it easier for agents to purchase homes. For concreteness we focus on the utility of owning a home. Suppose that before time zero the economy is in a steady state with no uncertainty, so $P_{t}=P$. At time zero agents learn that in each period, with small probability $\phi$, the value of $\varepsilon$ will change permanently to a new level $\varepsilon^{*}$. Agents agree about the value of $\phi$ but disagree about the probability distribution for $\varepsilon^{*}$. Agents do not receive information that is useful for updating their priors about the distribution of $\varepsilon^{*}{ }^{7}$ As soon as uncertainty is resolved agents become homogeneous in terms of their beliefs.

Prior to the resolution of uncertainty, agents fall into three categories depending on their priors about $\varepsilon^{*}$. Borrowing from the terminology used in the epidemiology literature we refer to these agents as "infected," "cured," and "vulnerable." We denote by $i_{t}, c_{t}$, and $v_{t}$ the time $t$ fraction of infected, cured and vulnerable agents, respectively. Agent types are indexed by $j=i, c, v$ and are assumed to be publicly observable. Priors are common knowledge, so higher-order beliefs play no role in our model. The laws of social dynamics described below are public information. Agents take into account future changes in the fractions of the population that hold different views.

\footnotetext{
${ }^{6}$ It is well known that there are explosive solutions to equation (3.1) (see, e.g. Diba and Grossman (1988)). We abstract from these solutions in our analysis.

${ }^{7}$ If agents disagreed about the value of $\phi$ they would update their priors about $\phi$ after observing whether a change in fundamentals occurred. We abstract from uncertainty about the value of $\phi$ to focus our analysis on the importance of social dynamics.
} 
The new value of the flow utility of owning a home, $\varepsilon^{*}$, is drawn from the set $\Phi$. For simplicity we assume that this set contains $n$ elements. An agent of type $j$ attaches the probability distribution function (pdf) $f^{j}\left(\varepsilon^{*}\right)$ to the elements of $\Phi$.

We assume that at time zero there is a very small fraction of cured and infected agents. Almost all agents are vulnerable, i.e. they have diffuse priors about future fundamentals. Infected agents expect an improvement in fundamentals:

$$
E^{i}\left(\varepsilon^{*}\right)>\varepsilon
$$

Cured and vulnerable agents do not expect fundamentals to improve:

$$
E^{c}\left(\varepsilon^{*}\right)=E^{v}\left(\varepsilon^{*}\right)=\varepsilon
$$

For now we assume that agents do not take into account that they might change their type as a result of social interactions. This assumption rules out the possibility that agents might take actions that are optimal only because they might change their type in the future. For example, a cured agent might buy a home, even though this action is not optimal given his current priors, in anticipation of the possibility that he could become infected in the future. We return to this issue at the end of this section.

We use the entropy of the probability distribution $f^{j}\left(\varepsilon^{*}\right)$ to measure the uncertainty of an agent's views,

$$
e^{j}=-\sum_{i=1}^{n} f^{j}\left(\varepsilon_{i}^{*}\right) \ln \left[f^{j}\left(\varepsilon_{i}^{*}\right)\right]
$$

The higher is the value of $e^{j}$, the greater is an agent's uncertainty about $\varepsilon^{*}$. This uncertainty is maximal when the pdf is uniform, in which case $e^{j}=\ln (n)$.

Agents meet randomly at the beginning of the period. When agent $l$ meets agent $j$, agent $j$ adopts the priors of agent $l$ with probability $\gamma^{l j}$. The value of $\gamma^{l j}$ depends on the ratio of the entropies of the agents' pdfs:

$$
\gamma^{l j}=\max \left(1-e^{l} / e^{j}, 0\right)
$$

This equation implies that a low-entropy agent will not adopt the prior of a high-entropy agent. In addition, it implies that the probability that a high-entropy agent adopts the priors of the low-entropy agent is decreasing in the ratio of the two entropies $\left(e^{l} / e^{j}\right)$. We adopt this formulation for two reasons. First, it strikes us as plausible. Second, it is consistent 
with evidence from the psychology literature that people are more persuaded by those who are confident (e.g. Price and Stone (2004) and Sniezek and Van Swol (2001)).

Throughout we assume that the entropy of the vulnerable agents exceeds the entropy of the cured and infected:

$$
\begin{aligned}
& e^{c}<e^{v} \\
& e^{i}<e^{v} .
\end{aligned}
$$

In addition, we make the natural assumption that most agents are vulnerable at time zero and that the initial number of infected and cured agents is small but identical: $i_{0}=c_{0}$.

The population dynamics in our model are similar to the dynamics in the infectious disease models of Bernoulli (1766) and Kermack and McKendrick (1927). ${ }^{8}$ We consider two cases to analyze the equilibrium in which either the cured or the infected agents have the lowest entropy. In both cases, if uncertainty is not resolved, the entire population converges to the view of the agent with the lowest entropy. The model generates a "fad" in the sense that the fraction of the population with a particular view rises and then falls.

Case 1 Here we assume that the pdf of the cured agents has the lowest entropy:

$$
e^{c}<e^{i}<e^{v}
$$

The fraction of the population with different views evolves according to:

$$
\begin{gathered}
i_{t+1}=i_{t}+\gamma^{i v} i_{t} v_{t}-\gamma^{c i} i_{t} c_{t}, \\
c_{t+1}=c_{t}+\gamma^{c v} c_{t} v_{t}+\gamma^{c i} i_{t} c_{t}, \\
v_{t+1}=v_{t}-\gamma^{i v} i_{t} v_{t}-\gamma^{c v} c_{t} v_{t} .
\end{gathered}
$$

To understand equation (3.4) note that there are $i_{t} v_{t}$ encounters between infected and vulnerable agents. ${ }^{9}$ As a result of these encounters, $\gamma^{i v} i_{t} v_{t}$ vulnerable agents become infected. Similarly, there are $c_{t} i_{t}$ encounters between cured and infected at time $t$. As a result of these encounters, $\gamma^{c i} c_{t} i_{t}$ infected agents become cured. These two sets of encounters, together with $i_{t}, c_{t}$, and $v_{t}$ determine $i_{t+1}$.

\footnotetext{
${ }^{8}$ Bernoulli (1766) used his model of the spread of smallpox to show that vaccination would result in a significant increase in life expectancy. When vaccination was introduced, insurance companies used Bernoulli's life-expectancy calculations to revise the price of annuity contracts (Dietz and Heesterbeek (2002)).

${ }^{9}$ See Duffie and Sun (2007) for a law of large numbers that applies to pairwise random meetings.
} 
Consider next equation (3.5). There are $i_{t} c_{t}$ encounters between cured and infected agents which lead to $\gamma^{c i} i_{t} c_{t}$ infected agents becoming cured. The are also $c_{t} v_{t}$ encounters between cured and vulnerable agents which lead to $\gamma^{c v} c_{t} v_{t}$ vulnerable agents becoming cured. Finally, equation (3.6) reflects the fact that $\gamma^{i v} i_{t} v_{t}$ vulnerable agents become infected and $\gamma^{c v} c_{t} v_{t}$ become cured.

In case 1 the model can generate a "fad" in which the number of infected agents rises for a while before declining towards zero. To see how this pattern emerges, suppose that initially a large fraction of the population is vulnerable and that $\gamma^{i v} v_{0}>\gamma^{c i} c_{0}$. In conjunction with equation (3.4) the latter condition implies that initially the fraction of infected agents grows over time.

Also, consider a path of the economy in which uncertainty is not realized. Along this path the number of infected agents initially rises over time as the number of vulnerable agents who become infected is larger than the number of infected agents who become cured (see equation (3.4)). The number of vulnerable agents falls over time as some of these agents become infected and others become cured (see equation (3.6)). Eventually, $\gamma^{i v} v_{t}<\gamma^{c i} c_{t}$. At this point the fraction of infected agents begins to fall. It follows from equation (3.5) that all agents become cured as $t \rightarrow \infty$.

Case 2 Here we assume that the pdf of the infected agents has the lowest entropy:

$$
e^{i}<e^{c}<e^{v}
$$

The fraction of the population with different views evolves according to:

$$
\begin{aligned}
& i_{t+1}=i_{t}+\gamma^{i v} i_{t} v_{t}+\gamma^{i c} i_{t} c_{t}, \\
& c_{t+1}=c_{t}+\gamma^{c v} c_{t} v_{t}-\gamma^{i c} i_{t} c_{t}, \\
& v_{t+1}=v_{t}-\gamma^{i v} i_{t} v_{t}-\gamma^{c v} c_{t} v_{t} .
\end{aligned}
$$

To understand equation (3.7) note that the $i_{t} v_{t}$ encounters between infected and vulnerable agents lead to $\gamma^{i v} i_{t} v_{t}$ vulnerable agents becoming infected. There are also $i_{t} c_{t}$ encounters between infected and cured which lead to $\gamma^{i c} i_{t} c_{t}$ cured agents becoming infected. In equation (3.8) the $i_{t} c_{t}$ encounters between cured and infected agents at time $t$ result in $\gamma^{i c} i_{t} c_{t}$ cured agents becoming infected. The $c_{t} v_{t}$ encounters between cured and vulnerable agents at time $t$ result in $\gamma^{c v} c_{t} v_{t}$ vulnerable agents become cured. Finally, equation (3.9) reflects the fact that $\gamma^{i v} i_{t} v_{t}$ vulnerable agents become infected and $\gamma^{c v} c_{t} v_{t}$ become cured. 
This version of the model also generates a "fad" but here it is the number of cured agents that rises for a while before declining towards zero. A fad arises when initially a large fraction of the population is vulnerable and $\gamma^{c v} v_{t}>\gamma^{i c} i_{t}$. The basic difference between case 1 and case 2 is that in the latter case cured agents are being converted into infected agents, so that eventually all agents become infected.

Equilibrium in the frictionless model House prices are determined by the marginal buyer. To determine the identity of this buyer we sort agents in declining order of how much they value houses. The marginal buyer is the agent who is at the kth percentile of house valuations. When the fraction of infected agents is lower than $k$ for all $t$, the marginal home buyer is a non-infected agent. Since these agents do not expect changes in the utility of owning a home, the price is constant over time at the value given by equation (3.2). In order to generate a boom-bust cycle, at least $k$ percent of the agents must be infected at some point in time.

It is useful to define the time-t fundamental value of a house before the resolution of uncertainty for a given agent, assuming that this agent is the marginal buyer until uncertainty is resolved. We denote these fundamental values for the infected, cured and vulnerable agents by $P_{t}^{i}, P_{t}^{v}$, and $P_{t}^{c}$, respectively. The value of $P_{t}^{i}$ is given by:

$$
P_{t}^{i}=\beta\left\{\phi\left[E^{i}\left(\varepsilon^{*}\right)+\beta \frac{E^{i}\left(\varepsilon^{*}\right)}{1-\beta}\right]+(1-\phi)\left(\varepsilon+P_{t+1}^{i}\right)\right\} .
$$

The logic that underlies this equation is as follows. With probability $\phi$ uncertainty is resolved. In this case the expected utility flow and house price at time $t+1$ are $E^{i}\left(\varepsilon^{*}\right)$ and $\beta E^{i}\left(\varepsilon^{*}\right) /(1-$ $\beta$ ), respectively. With probability $1-\phi$ uncertainty is not resolved. In this case the agent derives a utility flow, $\varepsilon$, and values the house at $P_{t+1}^{i}$. Since we are deriving the fundamental value under the assumption that the infected agent is always the marginal home buyer, $P_{t}^{i}=P_{t+1}^{i}=P^{i}$. Solving equation (3.10) for $P_{t}^{i}$ we obtain:

$$
P^{i}=\beta \frac{\phi E^{i}\left(\varepsilon^{*}\right) /(1-\beta)+(1-\phi) \varepsilon}{1-\beta(1-\phi)} .
$$

Vulnerable and cured agents expect $\varepsilon^{*}$ to equal $\varepsilon$, so:

$$
P^{c}=P^{v}=\beta \frac{\varepsilon}{1-\beta} .
$$

We begin by characterizing the equilibrium of the economy in case 1 . Suppose that the fraction of infected agents rises above $k$ for $t \in\left[t_{1}, t_{2}\right]$, where $t_{2}<\infty$. For $t>t_{2}$ the cured 
agents are the marginal home buyers. So, the price is given by:

$$
P_{t}=P^{c}, \text { for } t \geq t_{2}+1 \text {. }
$$

Using $P_{t_{2}+1}$ as a terminal value we can compute recursively the prices for $t \leq t_{2}$ that obtain if uncertainty is not realized. Since the infected agent is the marginal home buyer between period $t_{1}$ and period $t_{2}$ we have:

$$
P_{t}=\beta\left\{\phi\left[E^{i}\left(\varepsilon^{*}+P_{t+1}^{*}\right)\right]+(1-\phi)\left(\varepsilon+P_{t+1}\right)\right\}, \text { for } t_{1} \leq t \leq t_{2}
$$

Here $P_{t+1}$ and $P_{t+1}^{*}$ are the $t+1$ prices when uncertainty is not realized and when uncertainty is realized, respectively.

Since the vulnerable agents are the marginal home buyers for $t<t_{1}$, we have:

$$
P_{t}=\beta\left\{\phi\left[E^{c}\left(\varepsilon^{*}+P_{t+1}^{*}\right)\right]+(1-\phi)\left(\varepsilon+P_{t+1}\right)\right\}, \text { for } t<t_{1} .
$$

Here we use the fact that: $E^{c}\left(\varepsilon^{*}\right)=E^{v}\left(\varepsilon^{*}\right)$.

The following proposition characterizes the equilibrium for this model.

Proposition 3.1. The equilibrium price path in case 1 when uncertainty is not realized is given by:

$$
P_{t}= \begin{cases}P^{c}+[\beta(1-\phi)]^{t_{1}-t}\left(P_{t_{1}}-P^{c}\right), & t<t_{1} \\ P^{i}-[\beta(1-\phi)]^{t_{2}+1-t}\left(P^{i}-P^{c}\right), & t_{1} \leq t \leq t_{2} \\ P^{c}, & t>t_{2}\end{cases}
$$

The equilibrium price path when uncertainty is realized is given by:

$$
P_{t}=\frac{\beta \varepsilon^{*}}{1-\beta}
$$

The intuition for this proposition is as follows. Before time $t_{1}$ the marginal buyer is a vulnerable agent. If uncertainty is not realized, the marginal buyer at time $t_{1}$ is an infected agent. The latter agent is willing to buy the house at a value that exceeds $P^{c}$. The price of a house reflects this expected capital gain, $P_{t_{1}}-P^{c}$. This capital gain is realized with probability $(1-\phi)^{t_{1}-t}$. So the expected, discounted capital gain is given by: $[\beta(1-\phi)]^{t_{1}-t}\left(P_{t_{1}}-P^{c}\right)$. Therefore, the equilibrium price is equal to $P^{c}$ plus this capital gain (first line of equation (3.14)). Note that the price jumps at time zero from $P^{c}$ to $P^{c}+[\beta(1-\phi)]^{t_{1}}\left(P_{t_{1}}-P^{c}\right)$ because of the expected capital gains associated with the change in the marginal buyer at time $t_{1}$. As long as uncertainty is not realized, the price rises before time $t_{1}$ reflecting the fact that the expected, discounted capital gain is rising at rate $\beta(1-\phi)$. 
Between time $t_{1}$ and $t_{2}$ the marginal buyer is an infected agent. However, if uncertainty is not realized, the marginal buyer at time $t_{2}$ is a cured agent who is willing to buy the house at a price $P^{c}<P^{i}$. The equilibrium price is equal to $P^{i}$ minus the expected, discounted capital loss, $[\beta(1-\phi)]^{t_{2}-t}\left(P^{i}-P^{c}\right)$ (second line of equation (3.14)). As long as uncertainty is not realized, the price falls before $t_{2}+1$ reflecting the fact that the expected, discounted capital loss is rising at rate $\beta(1-\phi)$.

After time $t_{2}+1$ there are no more changes in the identity of the marginal buyer. So, unless uncertainty is realized, the price remains constant and equal to the fundamental value of a house to a cured agent, $P^{c}$.

Finally, once uncertainty is realized, agents have homogeneous expectations so all fundamental values coincide and the price of a house is given by equation (3.15).

Proposition 3.1 implies that the model generates a boom-bust cycle in house prices as long as uncertainty is not realized. Of course, the model can also generate a boom-bust as well as a boom-no-bust path depending upon when uncertainty is realized and the realization of $\varepsilon^{*}$. We view these possibilities as less interesting because of the difficulty of identifying observable fundamentals that covary with house prices.

The following proposition characterizes the equilibrium price path in case 2. Recall that, in this case, the fraction of the population that is infected converges monotonically to one. We define $t_{1}$ as the first time period at which $i_{t} \geq k$. The behavior of prices is summarized by the following proposition.

Proposition 3.2. The equilibrium price path in case 2 when uncertainty is not realized is given by:

$$
P_{t}= \begin{cases}P^{c}+[\beta(1-\phi)]^{t_{1}-t}\left(P^{i}-P^{c}\right), & t<t_{1}, \\ P^{i}, & t \geq t_{1} .\end{cases}
$$

The equilibrium price path when uncertainty is realized is given by:

$$
P_{t}=\frac{\beta \varepsilon^{*}}{1-\beta} .
$$

The intuition for this proposition is as follows. After time $t_{1}$, until uncertainty is resolved, the marginal home buyer is an infected agent. So, absent resolution of uncertainty the price is equal to $P^{i}$ for all $t \geq t_{1}$. Before $t_{1}$ the marginal home buyer is a vulnerable/cured agent who has a fundamental house value $P^{c}$. The equilibrium price is equal to $P^{c}$ plus the discounted expected value of the capital gain that results from selling the house to an infected agent at time $t_{1},[\beta(1-\phi)]^{t_{1}-t}\left(P^{i}-P^{c}\right)$. 
A simple numerical example We now consider a simple numerical example that illustrates the properties of the model summarized in the previous proposition. In case 1 equations (3.5)-(3.6) imply that the maximum value of $i_{t}$ is 50 percent. So, the presence of infected agents affects prices only if $k<0.5$. In the following example we assume that $k=0.1$.

We use the beta distribution to guide our choice of pdfs over $\varepsilon^{*}$ for the different agents in the economy. This family of continuous distributions, which depends on two parameters, $x$ and $y$, is very flexible and includes the uniform distribution as a particular case. We denote by $x^{j}$ and $y^{j}$ the parameters of the beta distribution of agent $j .^{10}$

To simplify our computations we work with a discrete approximation to the beta distributions defined on a symmetric grid with six points. To compute the probability of each of these points we divide the support of the distribution into six intervals of equal size and compute the integral of the beta distribution over these intervals. The support of the distribution corresponds to the mid points of the different intervals. ${ }^{11}$

Our choice of parameters $x^{j}$ and $y^{j}$ are such that the discretized beta distributions imply: $E^{v}\left(\varepsilon^{*}\right)=E^{c}\left(\varepsilon^{*}\right)=2.9, E^{i}\left(\varepsilon^{*}\right)=8.5$. We think of a time period as one month and choose $\beta$ so that the implied annual discount rate is six percent. We assume that there is a very small number of infected and cured natural renters at time zero: $i_{0}=c_{0}=10^{-5}$. The remainder of the population is vulnerable. We choose $\phi$, the probability that uncertainty is realized in each period, to equal 1/120. Absent resolution of uncertainty this value, together with our other assumptions, implies that a boom-bust pattern emerges over the course of roughly 20 years. Our value of $\phi$ implies that the probability of observing such a boom-bust pattern is roughly 13 percent.

Case 1 Here our choice of $x^{j}$ and $y^{j}$ imply:

$$
\begin{aligned}
e^{i} & =0.82, e^{c}=0.81, \text { and } e^{v}=0.93 \\
\gamma^{c i} & =0.010, \gamma^{i v}=0.116, \text { and } \gamma^{c v}=0.125
\end{aligned}
$$

Panel (a) of Figure 2 shows the evolution of the fraction of cured, infected and vulnerable agents absent resolution of uncertainty about $\varepsilon^{*}$. The fraction of infected agents in the population initially increases slowly. The infection then gathers momentum until the fraction of

\footnotetext{
${ }^{10} \mathrm{We}$ assume that the support of the distribution is the interval $[0,10]$. The pdf of infected, cured and vulnerable agents are parameterized by: $x^{i}=2.4, y^{i}=0.3, x^{c}=9.35, y^{c}=22.4, x^{v}=7.0$, and $y^{v}=16.8$.

${ }^{11}$ The support is given by: $\varepsilon^{*} \in\{0.83,2.50,4.17,5.83,7.50,9.17\}$.
} 
infected agents peaks at 29 percent in year eight. Thereafter this fraction declines eventually reaching zero. Between $t_{1}$, the middle of year eight, and $t_{2}$, the beginning of year 19 , the infected agents are the marginal buyers since they exceed a fraction $k$ percent of the population. The fraction of vulnerable agents falls over time and converges towards zero as these agents become either cured or infected. The fraction of cured agents rises monotonically over time until everybody in the economy is cured.

The intuition for these dynamics is as follows. Since the vulnerable agents have the highest entropy, both infected and cured agents can convert the vulnerable agents to their views. The entropy of the cured agents is only slightly lower than that of the infected agents. By assumption there are initially very few infected and cured agents. Since the probability of these agents meeting is very small, very few infected agents become cured. Initially, both the fraction of cured and infected agents grows because there is a large pool of vulnerable agents for them to convert. Eventually, the number of vulnerable agents falls and very few vulnerable agents became newly infected. Because there is a large pool of cured agents, the probability that an infected agent meets a cured agents becomes substantial. Consequently, the number of infected agents that become cured rises. With low inflows and high outflows the fraction of infected agents asymptotes to zero. So, in this case we observe a 'fad' in the sense that the number of infected agents initially rises and then declines.

Consistent with Proposition 3.1, Figure 3 shows that the price jumps at time zero and then continues to rise slowly until infected agents become the marginal home buyers at time $t_{1}$. Thereafter the price drops rapidly, reverting to its initial steady-state value.

Figure 3 also displays the one-period-ahead annualized rate of return that different agents expect at each point, conditional on uncertainty not being realized at time $t$ :

$$
r_{t}^{j}=\frac{\phi\left[E^{j}\left(\varepsilon^{*}\right)+\beta E^{j}\left(\varepsilon^{*}\right) /(1-\beta)\right]+(1-\phi)\left(\varepsilon+P_{t+1}\right)}{P_{t}}-1 .
$$

The Figure also displays the volume of transactions implied by the model computed under the assumption that trade only occurs when at least one of the agents has a motive for transacting.

A key feature of Figure 3 is that agents have heterogeneous beliefs about the expected rate of return to housing. This basic feature of our model is consistent with the findings in Piazzesi and Schneider (2009) who document such heterogeneity using data from the Michigan Survey of Consumers. ${ }^{12}$

\footnotetext{
${ }^{12}$ Vissing-Jørgensen (2003) provides evidence for substantial heterogeneity of beliefs regarding the returns
} 
The annualized real rate of return to the marginal home owner is constant and equal to six percent. Before $t_{1}$ the cured/vulnerable agents are the marginal home owners. Infected agents expect very high rates of return which reflects a high value of $E^{i}\left(\varepsilon^{*}\right)$. So all newly infected agents $\left(\gamma^{i v} i_{t} v_{t}\right)$ buy homes. ${ }^{13}$ During this period prices are rising and all transactions are initiated by agents who buy homes. Prices and transaction volume simultaneously peak at time $t_{1}$.

Between time $t_{1}$ and $t_{2}$ the infected agents are the marginal buyers. During this period of time the cured/vulnerable agents expect negative rates of return because they have a low expected value of $\varepsilon^{*}$. So all newly cured agents $\left(\gamma^{c i} c_{t} i_{t}\right)$ sell their homes to infected agents who are indifferent between buying and holding. During this period prices are falling and all transactions are initiated by agents who sell homes. Figure 3 displays the time series of transactions volume. Notice that prices and transactions volume simultaneously peak at time $t_{1}$. Transaction volume collapses once prices start to fall because at this point infected agents own all the houses. After time $t_{1}$ the number of transactions recovers as some infected agents become cured and sell their homes.

After time $t_{2}$ the cured/vulnerable agents are once again the marginal home owners and infected agents expect very high rates of return that are not reflected in market prices. So, $\gamma^{i v} i_{t} v_{t}$ agents buy homes. But, because there are so few vulnerable agents the number of transactions is close to zero.

From Figure 3 we see that, while the identity of the marginal home owner changes over time, the rate of return to the marginal owner is fixed and constant at six percent. There is heterogeneity in agents' expected rate of return to housing. However, since pricing is determined by the marginal owner, the expectations of inframarginal agents are not reflected in home prices.

Finally, Figure 3 displays the price paths expected by infected and cured/vulnerable agents at time zero. These paths are given by:

$$
E_{0}^{j}\left(P_{t}\right)=(1-\phi)^{t+1}\left(\varepsilon+P_{t+1}\right)+\left[1-(1-\phi)^{t+1}\right]\left[E^{j}\left(\varepsilon^{*}\right)+\beta E^{j}\left(\varepsilon^{*}\right) /(1-\beta)\right]
$$

for $j=i, c, v$.

to other assets such as stocks.

${ }^{13}$ We assume that the vulnerable agents sell since they are indifferent between holding and selling. The infected agents could induce them to sell by offerering an arbitrarially small premium. Some infected agents become cured during this time period. However, for $t \leq t_{1}$ infected agents who become cured are indifferent between holding and selling, so we assume that they do not transact. 
Infected agents expect prices to rise very rapidly until time $t_{1}$. Thereafter expected prices continue to rise but at a diminished rate reflecting the fall in actual market prices if uncertainty is not realized. The fall in market prices is outweighed by the large rise in $\varepsilon^{*}$ that infected agents expect. Finally, expected prices rise at a slightly higher rate after time $t_{2}$, reflecting the fact that the price remains constant if uncertainty is not realized.

Consider next the price path expected by cured and vulnerable agents at time zero. Equation (3.19) implies that:

$$
E_{0}^{i}\left(P_{t}\right)-E_{0}^{c}\left(P_{t}\right)=\left[1-(1-\phi)^{t+1}\right] \frac{E^{i}\left(\varepsilon^{*}\right)-E^{c}\left(\varepsilon^{*}\right)}{1-\beta} .
$$

So, the entire difference between $E_{0}^{i}\left(P_{t}\right)$ and $E_{0}^{c}\left(P_{t}\right)$ reflects agents' different expectations about $\varepsilon^{*}$. This difference implies that cured/vulnerable agents always expect a lower price than infected agents. The cured/vulnerable agents expect prices to rise between time zero and time $t_{1}$ because the price appreciation that occurs as long as uncertainty is not realized outweighs the fall in price that occurs if uncertainty is realized. Between time $t_{1}$ and $t_{2}$ the latter effect outweighs the first effect and the cured/vulnerable agents expect prices to fall. After time $t_{2}$ the market price corresponds to the cured/vulnerable agent's fundamental price so prices are expected to be constant.

Case 2 Here our choice of $x^{j}$ and $y^{j}$ implies: ${ }^{14}$

$$
\begin{aligned}
e^{i} & =0.81, e^{c}=0.82, \text { and } e^{v}=0.93 \\
\gamma^{i c} & =0.010, \gamma^{i v}=0.125, \text { and } \gamma^{c v}=0.116 .
\end{aligned}
$$

Panel (b) of Figure 2 shows the evolution of the fraction of cured, infected, and vulnerable agents absent resolution of uncertainty about $\varepsilon^{*}$. The dynamics are the same as in panel (a) of Figure 2 except that the cured and infected have changed places. Here there is a fad in the sense that the number of cured agents rises for roughly eight years before falling to zero. The fraction of infected agents rises monotonically over time until everybody in the economy is infected.

Consistent with Proposition 3.2, Figure 4 shows that the price jumps at time zero and then continues to rise until the beginning of year five, when all homes are owned by infected agents. Thereafter the price is constant and equal to the infected agent's fundamental value.

\footnotetext{
${ }^{14}$ The pdf of infected, cured and vulnerable agents are parameterized by: $x^{c}=2.4, y^{c}=0.3, x^{i}=9.35$, $y^{i}=22.4, x^{v}=7.0$, and $y^{v}=16.8$.
} 
Figure 4 also shows the volume of transactions implied by the model. At time zero all homes are owned by vulnerable agents. Between time zero and time $t_{1}$ all newly infected agents $\left(\gamma^{i v} i_{t} v_{t}\right)$ buy homes. At time $t_{1}$ all of the homes are owned by infected agents and there are no new transactions because infected agents do not become cured.

Finally, Figure 4 displays the price path expected by infected and cured/vulnerable agents at time zero. This path is computed using equation (3.19). Infected agents expect prices to rise very rapidly until $t_{1}$. From this point on the expected price continues to rise because the probability that uncertainty is realized and infected agents receive a large capital gain increases with the time horizon. Figure 4 also displays the price path expected by cured and vulnerable agents at time zero. As in case 1, the entire difference between $E_{0}^{i}\left(P_{t}\right)$ and $E_{0}^{c}\left(P_{t}\right)$ reflects different expectations about $\varepsilon^{*}$ (see equation (3.20)).

Interpreting cross-sectional data on housing prices A salient feature of the data on house prices is the presence of both boom-bust episodes and episodes in which booms are not followed by busts. Our model is consistent with this feature of the data. Regardless of whether we are in case 1 or case 2 , infected agents expect prices to rise and eventually level off, while cured agents expect prices to rise and then fall. An econometrician taking repeated samples from our data would see both boom-busts and booms that are not followed by busts. The boom-bust episodes would occur in economies where the cured agents happen to be correct. The booms that are not followed by busts would occur in economies where the infected agents happen to be right. Of course, in any given economy the econometrician cannot predict ex-ante which type of episode he would see. That is because by construction the data are not informative about which agent is correct.

An alternative interpretation of social dynamics We conclude this section by describing an alternative environment which generates social dynamics that are similar to those of our model. In this example agents have heterogeneous priors and receive private signals. Suppose that the agents who are initially infected and cured have very sharp priors. Agents that are initially vulnerable have very diffuse priors. All agents receive uninformative private signals. Vulnerable agents have sharp priors that the posteriors of infected and cured agents are the product of initially diffuse priors and very informative signals. So, when a vulnerable agent meets an infected (cured) agent his posterior becomes arbitrarily close to that of the infected (cured) agent. We refer to a vulnerable agent who has a posterior that is very close 
to that of an infected (cured) agent as infected (cured).

We reinterpret $\gamma^{l j}$ as the probability that agents of type $l$ meet agents of type $j$. We assume that $\gamma^{v c}=\gamma^{v i}=\gamma$ and that $\gamma^{c i}=0$, i.e. cured and infected agents have no social interactions. Under our assumptions the dynamics of the fraction of population with different views are similar to those generated by our model of social dynamics. Our assumptions about $\gamma^{l j}$ eliminate the convergence of posteriors that is a generic property of Bayesian environments. As a result, we preserve the property that different agents agree to disagree. ${ }^{15}$ To obtain dynamics similar to cases 1 and 2 we need to introduce a slight asymmetry between cured and infected agents. A simple, albeit mechanical, way to introduce this asymmetry is to suppose that in case 1 (case 2) a small fraction $\delta$ of infected (cured) agents exogenously change their view to those of cured (infected) agents.

The view of social segmentation embodied in our assumptions about $\gamma^{l j}$ is consistent with the notion that agents who are strongly committed to a point of view limit their interactions to sources of information and individuals that are likely to confirm their own views. This phenomenon is discussed by Sunstein (2001) and Mullainathan and Shleifer (2005). The latter authors summarize research in psychology, communications and information theory that is consistent with the social-segmentation hypothesis. More recently, Gentzkow and Shapiro (2010) find evidence that people tend to have close social interactions with people who have similar political views. Social segmentation is related to what sociologists call "homophily": contact between similar people occurs at a higher rate than contact among dissimilar people (McPherson, Smith-Lovin, Cook (2001)).

Internalizing changes in agent type So far we have assumed that agents do not take into account that they may change their type as a result of social interactions. Here we assess the quantitative impact of this assumption by calculating equilibrium prices when agents do internalize the possibility that they may change their type.

In case 1 , absent resolution of uncertainty, all agents become cured as $t$ goes to infinity and the terminal price is equal to the fundamental price of a cured agent $\left(P^{c}\right.$ in equation (3.12)). In case 2, absent resolution of uncertainty, all agents become infected as $t$ goes to infinity and the terminal price is equal to the fundamental price of an infected agent $\left(P^{i}\right.$ in equation (3.11)). Using these terminal prices we can compute the equilibrium price path in

\footnotetext{
${ }^{15}$ Acemoglu et al. (2007) provide an alternative environment in which agents agree to disagree because they are uncertain about the interpretation of the signals that they receive.
} 
a recursive fashion.

When $i(t) \geq k$ infected agents are the marginal home owners. In this case the equilibrium price is given by:

$$
\begin{gathered}
P_{t}=\left(1-\gamma^{c i} c_{t}\right) \beta\left[\phi E^{i}\left(\varepsilon^{*}+P_{t+1}^{*}\right)+(1-\phi)\left(\varepsilon+P_{t+1}\right)\right]+ \\
\gamma^{c i} c_{t} \beta\left[\phi E^{c}\left(\varepsilon^{*}+P_{t+1}^{*}\right)+(1-\phi)\left(\varepsilon+P_{t+1}\right)\right] .
\end{gathered}
$$

Recall that $P_{t+1}$ and $P_{t+1}^{*}$ are the $t+1$ prices when uncertainty is not realized and when uncertainty is realized, respectively. Here an infected agent takes into into account that with probability $\gamma^{c i} c_{t}$ he becomes cured at time $t+1$ and values the house as a cured agent. The value of $\gamma^{c i}$ is positive in case 1 but equal to zero in case 2 .

When $i(t)<k$ and $i(t)+v(t) \geq k$ vulnerable agents are the marginal home owners even if $c(t) \geq k$. Vulnerable agents have higher valuations than cured agents because they have a higher probability of becoming infected. In this case the equilibrium price is given by:

$$
\begin{aligned}
P_{t}=(1- & \left.\gamma^{i v} i_{t}-\gamma^{c v} c_{t}\right) \beta\left[\phi E^{v}\left(\varepsilon^{*}+P_{t+1}^{*}\right)+(1-\phi)\left(\varepsilon+P_{t+1}\right)\right] \\
+ & \gamma^{i v} i_{t} \beta\left[\phi E^{i}\left(\varepsilon^{*}+P_{t+1}^{*}\right)+(1-\phi)\left(\varepsilon+P_{t+1}\right)\right]+ \\
& +\gamma^{c v} c_{t} \beta\left[\phi E^{c}\left(\varepsilon^{*}+P_{t+1}^{*}\right)+(1-\phi)\left(\varepsilon+P_{t+1}\right)\right] .
\end{aligned}
$$

Here the vulnerable agent takes into account that with probability $\gamma^{i v} i_{t}$ he becomes infected and values the house as an infected agent. Also, with probability $\gamma^{c v} c_{t}$ he becomes cured and values the house as a cured agent.

Finally, when $i(t)<k$ and $i(t)+v(t)<k$ the marginal home owner is a cured agent. In this case the equilibrium price is given by:

$$
\begin{aligned}
& P_{t}=\gamma^{i c} i_{t} \beta\left[\phi E^{i}\left(\varepsilon^{*}+P_{t+1}^{*}\right)+(1-\phi)\left(\varepsilon+P_{t+1}\right)\right]+ \\
&\left(1-\gamma^{i c} i_{t}\right) \beta\left[\phi E^{c}\left(\varepsilon^{*}+P_{t+1}^{*}\right)+(1-\phi)\left(\varepsilon+P_{t+1}\right)\right] .
\end{aligned}
$$

Here the cured agent takes into account that, with probability $\gamma^{i c} i_{t}$ he becomes infected and values the house as an infected agent. Recall that $\gamma^{i c}$ is zero in case 1 but it is positive in case 2 .

We redo the experiment that underlies Figure 3 using the same parameter values. The basic finding is that internalizing changes in agent type makes virtually no difference to our results. The basic reason is that the probability of switching types is small. For instance, in case 1 the maximum value of $\gamma^{i v} i_{t}$ and $\gamma^{c i} c_{t}$ in our numerical example are three and one-third 
of one percent, respectively. In the following sections we abstract from this effect to simplify our computations.

\section{A matching model}

The frictionless model shows the potential of social dynamics to account for the house price dynamics observed in the data. However, it has three unattractive features. First, to generate a boom-bust cycle in case 1, the fraction of agents that are infected must exceed $k$ for at least some period of time. According to the Housing Vacancy Survey of the Bureau of the Census, the average fraction of American households who owned homes during the period 1965-2010 is 65 percent. So, the model requires that a very large fraction of the population become infected. ${ }^{16}$ Second, the price rise that occurs at time zero is large relative to the peak rise in prices. Third, the model is too stylized to account for the fact that the volume of transactions and time to sell are highly correlated with average housing prices (see Stein (1995)).

Here we model the housing market using an extended version of the matching model proposed by Piazzesi and Schneider (2009). In this section we consider a version of the model in which agents have homogeneous expectations. The basic structure of this model coincides with that of the frictionless model described in Section 3. The economy is populated by a continuum of agents with measure one. All agents have linear utility and discount utility at rate $\beta$. There is a fixed stock of houses, $k<1$, in the economy and a rental market with $1-k$ houses. Rental units are produced by competitive firms at a cost $w$ per period, so the rental rate is constant and equal to $w$.

There are four types of agents in the economy: homeowners, home sellers, natural home buyers, and natural renters. We denote the fraction of these agents in the population by $h_{t}, u_{t}, b_{t}$, and $r_{t}$, respectively. Home owners and home sellers own homes at time $t$. In equilibrium all homes are occupied so that:

$$
h_{t}+u_{t}=k .
$$

\footnotetext{
${ }^{16}$ An alternative strategy for remedying this shortcoming is to allow for heterogeneity in the utility of owning a home. For example, suppose there is a group of agents that would never sell their home because they derive such a high utility from it. The presence of this group is equivalent to a reduction in $k$, so that it is easier to generate a boom in the price of homes that are potentially for sale.
} 
Both natural buyers and natural renters rent homes at time $t$ so that:

$$
b_{t}+r_{t}=1-k
$$

We describe the state of the economy by $s_{t}=\left\{h_{t}, b_{t}\right\}$. We now discuss the problems faced by the different agents in the economy.

Homeowners A homeowner derives momentary utility $\varepsilon$ from his home. The agent's value function, $H\left(s_{t}\right)$, is given by:

$$
H\left(s_{t}\right)=\varepsilon+\beta\left[(1-\eta) H\left(s_{t+1}\right)+\eta U\left(s_{t+1}\right)\right] .
$$

With probability $\eta$ a homeowner's match with his home goes sour and he becomes a home seller. We denote the value function of a home seller by $U\left(s_{t}\right)$.

Home sellers A home seller sells his home with probability $p\left(s_{t}\right)$. Once the sale occurs, the home seller becomes a natural renter. The home seller's value function is given by:

$$
U\left(s_{t}\right)=p\left(s_{t}\right)\left[P\left(s_{t}\right)+\beta R\left(s_{t+1}\right)\right]+\left[1-p\left(s_{t}\right)\right] \beta U\left(s_{t+1}\right) .
$$

Here $P\left(s_{t}\right)$ is the expected price received by a home seller and $R\left(s_{t}\right)$ is the value function of a natural renter.

To simplify, we abstract from the transactions costs of selling a home. In addition, we assume that the reservation price of a home seller, $\bar{P}^{u}$, is an exogenous constant. To ensure that transactions occur in the steady state we require that $\bar{P}^{u}$ be lower than the steady state reservation price of natural buyers.

Natural home buyers A natural buyer is a renter at time $t$. He has to choose between renting at $t+1$ or trying to buy a home. His net flow utility from renting is given by $\varepsilon^{b}-w$ and his value function is given by $B\left(s_{t}\right)$. If he decides to continue renting, his value function, $B^{\text {rent }}\left(s_{t}\right)$, is given by:

$$
B^{r e n t}\left(s_{t}\right)=\varepsilon^{b}-w+\beta B\left(s_{t+1}\right)
$$

If he tries to buy a house, he succeeds with probability $q\left(s_{t}\right)$. In this case, he pays a price $P^{b}\left(s_{t}\right)$ and his continuation utility is that of a home owner $\left((1-\eta) H\left(s_{t+1}\right)+\eta U\left(s_{t+1}\right)\right)$. With 
probability $1-q\left(s_{t}\right)$, he does not succeed in buying a house and he remains a renter at time $t+1$. So the value function of being a potential buyer, $B^{\text {buy }}\left(s_{t}\right)$, is given by:

$$
\begin{gathered}
B^{\text {buy }}\left(s_{t}\right)=q\left(s_{t}\right)\left\{\varepsilon^{b}-w-P^{b}\left(s_{t}\right)+\beta\left[(1-\eta) H\left(s_{t+1}\right)+\eta U\left(s_{t+1}\right)\right]\right\} \\
+\left[1-q\left(s_{t}\right)\right] B^{r e n t}\left(s_{t}\right) .
\end{gathered}
$$

The value function of a natural home buyer is given by:

$$
B\left(s_{t}\right)=\max \left[B^{\text {rent }}\left(s_{t}\right), B^{\text {buy }}\left(s_{t}\right)\right]
$$

The reservation price, $\bar{P}^{b}\left(s_{t}\right)$, is the price that makes a natural buyer indifferent between buying and renting:

$$
\bar{P}^{b}\left(s_{t}\right)=\beta\left[(1-\eta) H\left(s_{t+1}\right)+\eta U\left(s_{t+1}\right)-B\left(s_{t+1}\right)\right] .
$$

Natural renters A natural renter is a renter at time $t$. His net flow utility from renting is given by $\varepsilon^{r}-w$ and his value function is $R\left(s_{t}\right)$. The only difference between natural renters and natural buyers is that the former derive lower utility from owning a home. We model this difference by assuming that whenever natural renters buy a house they pay a fixed cost, $\kappa \varepsilon$. This fixed cost represents the expected present value of the difference between their utility from owning a home and the corresponding utility of a natural buyer. ${ }^{17}$ We choose the value of $\kappa$ so that it is not optimal for natural renters to buy a house in the steady state.

In each period a fraction $\lambda$ of natural renters receive a preference shock and become natural home buyers. A natural renter can choose whether to continue renting or to try to buy a house. If he continues renting, his value function, $R^{r e n t}\left(s_{t}\right)$, is given by:

$$
R^{\text {rent }}\left(s_{t}\right)=\varepsilon^{r}-w+\beta\left[(1-\lambda) R\left(s_{t+1}\right)+\lambda B\left(s_{t+1}\right)\right] .
$$

The continuation utility reflects the fact that a natural renter becomes a natural home buyer with probability $\lambda$.

If the natural renter tries to buy a house, he succeeds with probability $q\left(s_{t}\right)$. In this case, he pays a price $P^{r}\left(s_{t}\right)$ and his continuation utility is the same as that of a home owner $\left((1-\eta) H\left(s_{t+1}\right)+\eta U\left(s_{t+1}\right)\right)$, except that he must pay the fixed cost $\kappa \varepsilon$. With probability

\footnotetext{
${ }^{17}$ Since the fixed cost is paid upfront all home owners are identical. It does not matter whether they used to be natural buyers or natural renters. This property simplifies our analysis by reducing the number of different agents in the economy.
} 
$1-q\left(s_{t}\right)$ the natural renter does not succeed in buying a house. In this case, he continues to be a renter at time $t+1$. The value function of a potential buyer, $R^{\text {buy }}\left(s_{t}\right)$, is given by:

$$
\begin{aligned}
R^{\text {buy }}\left(s_{t}\right)=q\left(s_{t}\right) & \left\{\varepsilon^{r}-w-P^{r}\left(s_{t}\right)-\kappa \varepsilon+\beta\left[(1-\eta) H\left(s_{t+1}\right)+\eta U\left(s_{t+1}\right)\right]\right\} \\
+ & \left(1-q\left(s_{t}\right)\right) R^{r e n t}\left(s_{t}\right) .
\end{aligned}
$$

The value function of a natural home buyer is given by:

$$
R\left(s_{t}\right)=\max \left[R^{\text {rent }}\left(s_{t}\right), R^{\text {buy }}\left(s_{t}\right)\right] .
$$

The reservation price, $\bar{P}^{r}\left(s_{t}\right)$, is the price that makes natural renters indifferent between buying and renting:

$$
\bar{P}^{r}\left(s_{t}\right)=\beta\left[(1-\eta) H\left(s_{t+1}\right)+\eta U\left(s_{t+1}\right)\right]-\beta\left[(1-\lambda) R\left(s_{t+1}\right)+\lambda B\left(s_{t+1}\right)\right]-\kappa \varepsilon .
$$

Timing The timing of events within a period is as follows. Preference shocks occur in the beginning of the period. With probability $\eta$ home owners become home sellers. With probability $\lambda$ natural renters become natural buyers. Transactions occur at the end of the period. A fraction $p\left(s_{t}\right)$ of home sellers sell their home while a fraction $q\left(s_{t}\right)$ of home buyers buy a house.

We define the indicator function $J^{b}\left(s_{t}\right)$ to take the value one if it is optimal for natural buyers to buy a house when the state of the economy is $s_{t}$ and zero otherwise. The indicator function $J^{r}\left(s_{t}\right)$ is equal to one if it is optimal for natural renters to buy a house when the state of the economy is $s_{t}$ and is equal to zero otherwise.

The laws of motion for the fraction of home owners, home sellers, natural home buyers and natural renters in the population are given by:

$$
\begin{gathered}
h_{t+1}=(1-\eta) h_{t}+q\left(s_{t}\right)\left[\left(b_{t}+\lambda r_{t}\right) J^{b}\left(s_{t}\right)+r_{t}(1-\lambda) J^{r}\left(s_{t}\right)\right] \\
u_{t+1}=\left(u_{t}+\eta h_{t}\right)\left(1-p\left(s_{t}\right)\right) \\
b_{t+1}=\left(b_{t}+\lambda r_{t}\right)\left[1-q\left(s_{t}\right) J^{b}\left(s_{t}\right)\right] \\
r_{t+1}=(1-\lambda) r_{t}\left[1-q\left(s_{t}\right) J^{r}\left(s_{t}\right)\right]+p\left(s_{t}\right)\left(u_{t}+\eta h_{t}\right) .
\end{gathered}
$$


The matching technology There is a technology that governs matches between buyers and sellers. Since agents can only own one home, only natural renters and natural buyers can potentially buy homes:

$$
\operatorname{Buyers}\left(s_{t}\right)=\left(b_{t}+\lambda r_{t}\right) J^{b}\left(s_{t}\right)+r_{t}(1-\lambda) J^{r}\left(s_{t}\right)
$$

There is no short selling and homeowners only sell when the match with their home goes sour. It follows that the fraction of the population that are sellers is given by:

$$
\operatorname{Sellers}\left(s_{t}\right)=u_{t}+\eta h_{t}
$$

When a match occurs, the transactions price is determined by generalized Nash bargaining. The bargaining power of buyers and sellers is $\theta$ and $1-\theta$, respectively. Matches can occur between a seller and a natural buyer or a natural renter. In the first case the price paid by the natural buyer, $P^{b}\left(s_{t}\right)$, is:

$$
P^{b}\left(s_{t}\right)=\theta \bar{P}^{b}\left(s_{t}\right)+(1-\theta) \bar{P}^{u} .
$$

In the second case, the price paid by the natural renter, $P^{r}\left(s_{t}\right)$, is:

$$
P^{r}\left(s_{t}\right)=\theta \bar{P}^{r}\left(s_{t}\right)+(1-\theta) \bar{P}^{u} .
$$

The average price received by a home seller, $P\left(s_{t}\right)$, is given by:

$$
P\left(s_{t}\right)=\frac{\left(b_{t}+\lambda r_{t}\right) J^{b}\left(s_{t}\right) P^{b}\left(s_{t}\right)+r_{t}(1-\lambda) J^{r}\left(s_{t}\right) P^{r}\left(s_{t}\right)}{\left(b_{t}+\lambda r_{t}\right) J^{b}\left(s_{t}\right)+r_{t}(1-\lambda) J^{r}\left(s_{t}\right)} .
$$

The number of homes sold, $m\left(s_{t}\right)$, is determined by the matching function:

$$
m\left(s_{t}\right)=\mu \operatorname{Sellers}\left(s_{t}\right)^{\alpha} \operatorname{Buyers}\left(s_{t}\right)^{1-\alpha} .
$$

The probabilities of selling $\left(p\left(s_{t}\right)\right)$ and buying $\left(q\left(s_{t}\right)\right)$ a house are given by:

$$
\begin{aligned}
& p\left(s_{t}\right)=m\left(s_{t}\right) / \operatorname{Sellers}\left(s_{t}\right), \\
& q\left(s_{t}\right)=m\left(s_{t}\right) / \operatorname{Buyers}\left(s_{t}\right) .
\end{aligned}
$$

\subsection{Solution Algorithm}

In this subsection we discuss our algorithm for solving the model. We begin with the steady state and then show how to solve the model given arbitrary initial conditions. 
Steady State It can be shown that the model economy has a unique steady state in which the fraction of the different types of agents is constant. We now solve for the steady-state values of the probability of buying and selling a home ( $p$ and $q$ ) and the fraction of the different agents in the population $(h, u, b$, and $r)$.

We choose values for the parameters $\mu, \lambda$ and $k$. We choose a value for $\eta$ so that the probabilities of buying and selling a house coincide in the steady state:

$$
p=q
$$

Equations (4.23) and (4.22) imply that $p=q=\mu$. This fact, together with equations (4.2) and (4.15) imply that the steady state number of natural buyers is given by:

$$
b=\frac{(1-\mu) \lambda(1-k)}{\mu+\lambda(1-\mu)} .
$$

We then solve for $r, u$, and $h$, as functions of $\mu, \lambda$ and $k$ using (4.2), (4.14) and (4.1).

The fact that $p=q$ implies that the number of buyers is equal to the number of sellers (equations (4.23) and (4.24)). Since there are $u+\eta r$ home sellers and $b+\lambda r$ buyers in the steady state we set $\eta$ so that:

$$
u+\eta h=b+\lambda r
$$

Given the values of $p$ and $q$ we can now solve for the steady-state values of the purchase price, the reservation price of a buyer, and the value functions of the different agents evaluated in the steady state: $P, P^{b}, H, U, B$ and $R$. To do so we use the steady state versions of equations (4.3), (4.4), (4.5), (4.6), (4.8), (4.9), (4.19), (4.20), and (4.21) and the fact that in the steady state $B=B^{\text {buy }}$ and $R=R^{\text {rent }}$.

Transitional Dynamics We assume that at time $T=2000$ the system has converged to the steady state. Consequently, we obtain an approximate solution because it takes an infinite number of periods for the model economy to converge to the steady state.

Let the set $S$ denote all the values of the state variable $s_{t}$ that occur along the transition path. First, guess that $J^{b}\left(s_{t}\right)=1$ and $J^{r}\left(s_{t}\right)=0$ for all $s_{t} \in S$. Second, using the initial conditions $s_{0}=\left\{h_{0}, b_{0}\right\}$ and equations (4.13) through (4.16), compute the sequence of values of $h_{t}, u_{t}, b_{t}$, and $r_{t}$. Third, use equations (4.17), (4.18), (4.23), (4.22), and (4.24) to compute the values of $p\left(s_{t}\right)$ and $q\left(s_{t}\right)$ for $s_{t} \in S$. Fourth, assume that: $H\left(s_{T}\right)=H$, $U\left(s_{T}\right)=U, B\left(s_{T}\right)=B$, and $R\left(s_{T}\right)=R$. Then use equations (4.3) to (4.12) and (4.21) 
to solve backwards for $\left\{H\left(s_{t}\right), U\left(s_{t}\right), B\left(s_{t}\right), R\left(s_{t}\right), P\left(s_{t}\right)\right\}$ for $t=1$ to $T$. Finally, verify whether the guesses for $J^{b}\left(s_{t}\right)$ and $J^{r}\left(s_{t}\right)$ for $s_{t} \in S$ describe the optimal behavior of buyers and sellers along the proposed transition path. If not, revise the guesses for $J^{b}\left(s_{t}\right)$ and $J^{r}\left(s_{t}\right)$ until a consistent solution is obtained.

\subsection{Experiments}

We illustrate the properties of the model through a series of experiments.

An expected improvement in fundamentals We first consider the same experiment that we study in the frictionless model but with homogeneous beliefs. At time zero agents suddenly anticipate that, with probability $\phi$, the utility of owning a home rises from $\varepsilon$ to $\varepsilon^{*}>\varepsilon$. It is easy to show that there are no transition dynamics and the economy converges immediately to a new steady state with a higher price. So, when beliefs are homogeneous, anticipated future changes in fundamentals are immediately reflected in today's price. Matching frictions per se do not produce interesting price dynamics, at least in the experiment studied here.

Transitional dynamics We now study an experiment that highlights the effect of an exogenous increase in the number of buyers on home prices. The resulting intuition is useful for understanding the results that we obtain when we incorporate social dynamics into the model. Suppose that the fraction of natural buyers in the population is initially higher than its steady state value, $b_{0}>b$. Since $r_{0}=1-k-b_{0}$, the fraction of natural renters in the population is initially lower than its steady state value. We denote by $s$ the steady state value of the state variables: $s=\{b, h\}$ and by $s_{0}=\left\{b_{0}, h\right\}$ the time-zero value of the state variables. Equations (4.22), (4.23) and (4.24) imply that the time-zero probability of buying a house is lower than it is in steady state: $q\left(s_{0}\right)<q(s)$. The time-zero probability of selling is higher than it is in steady state: $p\left(s_{0}\right)>p(s)$.

To illustrate the transition dynamics of the model we consider a numerical example based on parameter values summarized in Table 1 . We use the same values for $\beta, w$, and $\varepsilon$ used in Section 3. We set the stock of houses, $k$, equal to 0.65 , which coincides with the average fraction of homeowners in the United States over the period 1965 to 2010 . We set $\varepsilon^{r}, \varepsilon^{b}$, and $w$ to one. We set $\lambda$ to 0.02. This value of $\lambda$, together with the values of $\mu$ and $k$ and the assumption that the probability of buying and selling a home are the same in the steady 
state, implies that $\eta$ is equal to 0.0102. This value of $\eta$ implies that home owners sell their house on average every eight years. We set the matching function parameter $\mu$ to 0.13 . This value implies that the average time to sell a house in the steady state is 7.5 months. We set the matching parameter $\alpha$ and the bargaining parameter $\theta$ to 0.5 so as to treat buyers and sellers symmetrically. We set the reservation price of the seller to $\bar{P}^{u}=1$. This value is lower than the steady state reservation price of natural buyers, so that it is optimal for natural buyers to buy in the steady state. Finally, we set $\kappa=42$, a value which implies that the steady state utility of a natural renter who buys a home is 20 percent lower than that of a natural home buyer. Our assumptions imply that it is not optimal for natural renters to buy homes in the steady state.

We assume that the number of natural buyers at time zero is 50 percent above its steady state level. Figure 5 depicts the model's transition dynamics. Home prices are initially high and converge to the steady state from above. In addition, the paths for prices and the number of buyers mirror each other closely. To understand these properties notice that the utility of a home seller converges to the steady state from above $\left(U\left(s_{t+1}\right)>U(s)\right)$, a result that reflects two forces. First, because the number of buyers is high during the transition, the probability of selling is higher than in the steady state. Second, the price received by the seller is higher than in the steady state.

We now discuss the intuition for why $P\left(s_{t}\right)>P(s)$. Along the transition path only natural buyers want to buy houses, so the transactions price, $P\left(s_{t}\right)$ is given by:

$$
P\left(s_{t}\right)=\theta \bar{P}^{b}\left(s_{t}\right)+(1-\theta) \bar{P}^{u} .
$$

Since $\bar{P}^{u}$ is exogenous, movements in $P\left(s_{t}\right)$ are determined by movements in $\bar{P}^{b}\left(s_{t}\right)$. Equation (4.8) implies that $\bar{P}^{b}\left(s_{t}\right)$ is an increasing function of $H\left(s_{t+1}\right)$ and $U\left(s_{t+1}\right)$ and a decreasing function of $B\left(s_{t+1}\right)$. Since $U\left(s_{t+1}\right)$ is greater than $U(s)$, equation (4.3) implies that $H\left(s_{t+1}\right)>$ $H(s)$. In addition, the value function of a natural buyer approaches the steady state from below. The basic reason for why $B\left(s_{t+1}\right)<B(s)$ is that the probability of realizing the surplus from buying a home is low along the transition to the steady state $\left(q\left(s_{t}\right)<q(s)\right)$. Since $H\left(s_{t+1}\right)$ and $U\left(s_{t+1}\right)$ are above the steady state and $B\left(s_{t+1}\right)$ is below its steady state value, it follows from equation (4.8) that the reservation price must be above its steady state value, $\bar{P}^{b}\left(s_{t}\right)>\bar{P}^{b}(s)$.

In summary, in this experiment an increase in the initial number of buyers reduces the probability of buying a house and raises the probability of selling a house. In addition, it 
lowers the utility of buyers, raises the utility of sellers, and generates prices that are above their steady state value.

These results suggest that a boom-bust episode occurs if, for some reason, there is a persistent increase in the number of buyers followed by a persistent decrease. In the next section we show that social dynamics can generate the required movements in the number of buyers without observable movements in fundamentals.

\section{A matching model with social dynamics}

In this section we consider an economy that incorporates the social dynamics described in Section 3 into the model with matching frictions described in Section 4. We use this model to study the same basic experiment considered in Section 3. Suppose that before time zero the economy is in a steady state with no uncertainty. At time zero agents learn that, with a small probability $\phi$, the value of $\varepsilon$ will change permanently to a new level $\varepsilon^{*}$. Agents agree about the value of $\phi$ but disagree about the probability distribution for $\varepsilon^{*}$. Agents receive no information that is useful for updating their priors about the distribution of $\varepsilon^{*}$. Once uncertainty is resolved agents become homogeneous in terms of their beliefs. At that point the economy coincides with the one studied in the previous section where the utility of owning a home is $\varepsilon^{*}$. The economy then converges to a steady state from initial conditions that are determined by social dynamics and the timing of the resolution of uncertainty.

Agents' expectations about $\varepsilon^{*}$ depend on whether they are infected, cured or vulnerable. In addition agents can be home owners, home sellers, natural buyers, or natural renters. So, all told there are twelve different types of agents in the economy. We use the variables $h_{t}^{j}$, $u_{t}^{j}, b_{t}^{j}$, and $r_{t}^{j}$ to denote the fraction of the population of type $j$ agents who are homeowners, home sellers, natural home buyers, and natural renters, respectively. The index $j$ denotes whether the agent is infected, cured or vulnerable: $j \in\{i, c, v\}$.

As in Section 4, agents are subject to preference shocks which can turn natural renters into natural buyers and home owners into home sellers. The timing of events within a period is as follows. First, uncertainty about $\varepsilon^{*}$ is realized or not. Second, preference shocks occur. With probability $\eta$ home owners become home sellers. With probability $\lambda$ natural renters become natural buyers. Third, social interactions occur and agents potentially change their views. Fourth, transactions occur. 
Population dynamics To solve the model we must keep track of the fraction of the different types of agents in the model. To streamline our exposition we focus here on the law of motion for the fraction of natural renters who are vulnerable. In the appendix we describe the population dynamics for the other agents in the economy. The mechanics of these dynamics are similar to those which we now describe.

We denote the fraction of vulnerable natural renters at the beginning of the period, after preference shocks occur, after social interactions occur, and after purchases and sales occur, by $r_{t}^{v},\left(r_{t}^{v}\right)^{\prime},\left(r_{t}^{v}\right)^{\prime \prime}$, and $r_{t+1}^{v}$, respectively. At the beginning of the period, a fraction $\lambda$ of the natural renters become natural buyers,

$$
\left(r_{t}^{v}\right)^{\prime}=r_{t}^{v}(1-\lambda) .
$$

Next, social interactions occur. A fraction $\gamma^{c v} c_{t}$ of the vulnerable natural renters become cured and a fraction $\gamma^{i v} i_{t}$ become infected. Consequently, the fraction of vulnerable natural renters after social interactions is given by:

$$
\left(r_{t}^{v}\right)^{\prime \prime}=\left(r_{t}^{v}\right)^{\prime}-\gamma^{c v}\left(r_{t}^{v}\right)^{\prime} c_{t}-\gamma^{i v}\left(r_{t}^{v}\right)^{\prime} i_{t} .
$$

Transactions occur at the end of the period. Let $\left(u_{t}^{v}\right)^{\prime \prime}$ denote the fraction of the vulnerable natural sellers that remain after social interactions occur. All of these agents put their homes up for sale but only a fraction $p\left(s_{t}\right)$ succeed in actually selling their home. So the total number of sellers is $p\left(s_{t}\right)\left(u_{t}^{v}\right)^{\prime \prime}$. These sellers become natural renters. Let $J^{r, v}\left(s_{t}\right)$ denote an indicator function that is equal to one if it is optimal for a vulnerable natural renter to buy a home when the state of the economy is $s_{t}$ and zero otherwise. The number of vulnerable natural renters who try to purchase a home is equal to: $J^{r, v}\left(s_{t}\right)\left(r_{t}^{v}\right)^{\prime \prime}$. A fraction $q\left(s_{t}\right)$ of these agents succeed and become natural home owners. So, the number of vulnerable natural renters at the beginning of time $t+1$ is given by:

$$
r_{t+1}^{v}=\left(r_{t}^{v}\right)^{\prime \prime}-q\left(s_{t}\right) J^{r, v}\left(s_{t}\right)\left(r_{t}^{v}\right)^{\prime \prime}+p\left(s_{t}\right)\left(u_{t}^{v}\right)^{\prime \prime} .
$$

We now describe the value functions of the different agents in the economy. We begin by displaying the value functions that are relevant after uncertainty about $\varepsilon^{*}$ is realized. We then discuss the value functions that are relevant before $\varepsilon^{*}$ is realized.

Value functions after the realization of uncertainty We use upper bars to denote the value functions that apply after the resolution of uncertainty. These value functions depend 
on the realized value of $\varepsilon^{*}$ and on the state of the economy, $s_{t}$. Since the number of home owners adds up to $k$ and the number of renters to $1-k$, we can summarize the state of the economy by using ten of the 12 population fractions:

$$
s_{t}=\left\{b_{t}^{v}, b_{t}^{c}, b_{t}^{i}, r_{t}^{v}, r_{t}^{c}, h_{t}^{v}, h_{t}^{c}, h_{t}^{i}, u_{t}^{v}, u_{t}^{c}\right\}
$$

Let $\bar{H}\left(\varepsilon^{*}, s_{t}\right), \bar{U}\left(\varepsilon^{*}, s_{t}\right), \bar{B}\left(\varepsilon^{*}, s_{t}\right)$, and $\bar{R}\left(\varepsilon^{*}, s_{t}\right)$ denote the value function of a home owner, home seller, a natural buyer, and a natural renter, respectively. In addition, $P\left(\varepsilon^{*}, s_{t}\right)$, $P^{b}\left(\varepsilon^{*}, s_{t}\right)$, and $P^{r}\left(\varepsilon^{*}, s_{t}\right)$ denote the average price received by home sellers, the price paid by natural home buyers and the price paid by natural renters, respectively.

The value functions of home owners and home sellers are given by:

$$
\begin{gathered}
\bar{H}\left(\varepsilon^{*}, s_{t}\right)=\varepsilon^{*}+\beta\left[(1-\eta) \bar{H}\left(\varepsilon^{*}, s_{t+1}\right)+\eta \bar{U}\left(\varepsilon^{*}, s_{t+1}\right)\right] \\
\bar{U}\left(\varepsilon^{*}, s_{t}\right)=p\left(s_{t}\right)\left[P\left(\varepsilon^{*}, s_{t}\right)+\beta \bar{R}\left(\varepsilon^{*}, s_{t+1}\right)\right]+\left[1-p\left(s_{t}\right)\right] \beta \bar{U}\left(\varepsilon^{*}, s_{t+1}\right) .
\end{gathered}
$$

We denote by $\bar{B}^{\text {rent }}\left(\varepsilon^{*}, s_{t}\right)$ and $\bar{B}^{\text {buy }}\left(\varepsilon^{*}, s_{t}\right)$ the value function of a natural buyer who rents and buys, respectively. These functions can be written as:

$$
\begin{aligned}
\bar{B}^{\text {rent }}\left(\varepsilon^{*}, s_{t}\right)= & \varepsilon^{b}-w+\beta \bar{B}\left(\varepsilon^{*}, s_{t+1}\right) \\
\bar{B}^{\text {buy }}\left(\varepsilon^{*}, s_{t}\right)=q\left(s_{t}\right) & \left\{\varepsilon^{b}-w-P^{b}\left(\varepsilon^{*}, s_{t}\right)+\beta\left[(1-\eta) \bar{H}\left(\varepsilon^{*}, s_{t+1}\right)+\eta \bar{U}\left(\varepsilon^{*}, s_{t+1}\right)\right]\right\} \\
& +\left[1-q\left(s_{t}\right)\right] \bar{B}^{\text {rent }}\left(\varepsilon^{*}, s_{t}\right) .
\end{aligned}
$$

The value function $\bar{B}\left(\varepsilon^{*}, s_{t}\right)$ is given by:

$$
\bar{B}\left(\varepsilon^{*}, s_{t}\right)=\max \left[\bar{B}^{\text {rent }}\left(\varepsilon^{*}, s_{t}\right), \bar{B}^{\text {buy }}\left(\varepsilon^{*}, s_{t}\right)\right] .
$$

We denote by $\bar{R}^{r e n t}\left(\varepsilon^{*}, s_{t}\right)$ and $\bar{R}^{b u y}\left(\varepsilon^{*}, s_{t}\right)$ the value function of a natural renter associated with renting and buying, respectively. These functions can be written as:

$$
\begin{gathered}
\bar{R}^{\text {rent }}\left(\varepsilon^{*}, s_{t}\right)=\varepsilon^{r}-w+\beta\left[(1-\lambda) \bar{R}\left(\varepsilon^{*}, s_{t+1}\right)+\lambda \bar{B}\left(\varepsilon^{*}, s_{t+1}\right)\right], \\
\bar{R}^{\text {buy }}\left(\varepsilon^{*}, s_{t}\right)=q\left(s_{t}\right)\left\{\varepsilon^{r}-w-P^{r}\left(\varepsilon^{*}, s_{t}\right)-\kappa \varepsilon^{*}+\beta\left[(1-\eta) \bar{H}\left(\varepsilon^{*}, s_{t+1}\right)+\eta \bar{U}\left(\varepsilon^{*}, s_{t+1}\right)\right]\right\} \\
+\left[1-q\left(s_{t}\right)\right] \bar{R}^{\text {rent }}\left(\varepsilon^{*}, s_{t}\right) .
\end{gathered}
$$

In the previous equation we assume that the fixed cost $\left(k \varepsilon^{*}\right)$ paid by the natural renter for buying a home is proportional to the realized vale of $\varepsilon^{*}$. This assumption ensures that it is 
optimal for a natural renter to rent a home in the steady state of the economy regardless of the realized value of $\varepsilon^{*}$. The value function $\bar{R}\left(\varepsilon^{*}, s_{t}\right)$ is given by:

$$
\bar{R}\left(\varepsilon^{*}, s_{t}\right)=\max \left[\bar{R}^{r e n t}\left(\varepsilon^{*}, s_{t}\right), \bar{R}^{\text {buy }}\left(\varepsilon^{*}, s_{t}\right)\right] .
$$

The reservation prices of natural buyers and renters and the average transaction price are computed as in Section 4 using equations (4.8), (4.12), (4.19), (4.20), and (4.21). However, these equations must be modified by replacing $\varepsilon$ with $\varepsilon^{*}$.

Value functions before the realization of uncertainty Let $H^{j}\left(s_{t}\right), U^{j}\left(s_{t}\right), B^{j}\left(s_{t}\right)$, and $R^{j}\left(s_{t}\right)$ denote the value functions before uncertainty is realized of a type $j$ home owner, home seller, natural buyer and natural renter, respectively. In addition, $P\left(s_{t}\right), P^{b, j}\left(s_{t}\right)$, and $P^{r, j}\left(s_{t}\right)$ denote the average price received by home sellers, the average price paid by natural home buyers and the average price paid by natural renters, respectively.

The expectations operator $E^{j}\left[V^{j}\left(s_{t+1}\right)\right]$ denotes the expectation of a generic value function $V^{j}\left(s_{t+1}\right)$ based on the pdf of a type $j$ agent:

$$
E^{j}\left[V^{j}\left(s_{t+1}\right)\right]=(1-\phi) V^{j}\left(s_{t+1}\right)+\phi \sum_{\varepsilon^{*} \in \Phi} f^{j}\left(\varepsilon^{*}\right) \bar{V}\left(\varepsilon^{*}, s_{t+1}\right)
$$

Here $\bar{V}\left(\varepsilon^{*}, s_{t+1}\right)$ denotes the value function after the realization of uncertainty.

The value functions of a type $j$ home owner and home seller are given by:

$$
\begin{gathered}
H^{j}\left(s_{t}\right)=\varepsilon+\beta E^{j}\left[(1-\eta) H^{j}\left(s_{t+1}\right)+\eta U^{j}\left(s_{t+1}\right)\right] \\
U^{j}\left(s_{t}\right)=p\left(s_{t}\right)\left\{P\left(s_{t}\right)+\beta E^{j}\left[R^{j}\left(s_{t+1}\right)\right]\right\}+\left[1-p\left(s_{t}\right)\right] \beta E^{j}\left[U^{j}\left(s_{t+1}\right)\right] .
\end{gathered}
$$

We denote by $B^{r e n t, j}\left(s_{t}\right)$ and $B^{b u y, j}\left(s_{t}\right)$ the value function of a natural buyer of type $j$ associated with renting and buying, respectively. These functions can be written as:

$$
\begin{aligned}
B^{\text {rent }, j}\left(s_{t}\right)= & \varepsilon^{b}-w+\beta E^{j}\left[B^{j}\left(s_{t+1}\right)\right], \\
B^{\text {buy }, j}\left(s_{t}\right)= & q\left(s_{t}\right)\left\{\varepsilon^{b}-w-P^{b, j}\left(s_{t}\right)+\beta E^{j}\left[(1-\eta) H^{j}\left(s_{t+1}\right)+\eta U^{j}\left(s_{t+1}\right)\right]\right\} \\
& +\left[1-q\left(s_{t}\right)\right] B^{r e n t, j}\left(s_{t}\right) .
\end{aligned}
$$

The value function of a type $j$ natural buyer, $B^{j}\left(s_{t}\right)$, is given by:

$$
B^{j}\left(s_{t}\right)=\max \left[B^{r e n t, j}\left(s_{t}\right), B^{b u y, j}\left(s_{t}\right)\right] .
$$


The reservation price of a natural buyer of type $j, \bar{P}^{b, j}\left(s_{t}\right)$, is given by:

$$
\bar{P}^{b, j}\left(s_{t}\right)=E^{j} \beta\left[(1-\eta) H^{j}\left(s_{t+1}\right)+\eta U^{j}\left(s_{t+1}\right)-B^{j}\left(s_{t+1}\right)\right] .
$$

Recall that this price makes the agent indifferent between buying and renting.

We denote by $R^{r e n t, j}\left(s_{t}\right)$ and $R^{b u y, j}\left(s_{t}\right)$ the value function of a type $j$ natural renter who rents and buys, respectively. These functions can be written as:

$$
\begin{aligned}
R^{\text {rent }, j}\left(s_{t}\right)= & \varepsilon^{r}-w+\beta E^{j}\left\{(1-\lambda) R^{j}\left(s_{t+1}\right)+\lambda B^{j}\left(s_{t+1}\right)\right\} \\
R^{\text {buy }, j}\left(s_{t}\right)= & q\left(s_{t}\right)\left\{\varepsilon^{r}-w-P^{r, j}\left(s_{t}\right)-\kappa \varepsilon+\beta E^{j}\left[(1-\eta) H^{j}\left(s_{t+1}\right)+\eta U^{j}\left(s_{t+1}\right)\right]\right\} \\
& +\left[1-q\left(s_{t}\right)\right] R^{r e n t, j}\left(s_{t}\right) .
\end{aligned}
$$

The value function of a type $j$ natural renter, $R^{j}\left(s_{t}\right)$, is given by:

$$
R^{j}\left(s_{t}\right)=\max \left[R^{\text {rent }, j}\left(s_{t}\right), R^{b u y, j}\left(s_{t}\right)\right]
$$

The reservation price of a natural buyer of type $j, \bar{P}^{r, j}\left(s_{t}\right)$, is given by:

$$
\begin{aligned}
\bar{P}^{r, j}\left(s_{t}\right)=E^{j} \beta\{ & (1-\eta) H^{j}\left(s_{t+1}\right)+\eta U^{j}\left(s_{t+1}\right) \\
& \left.-(1-\lambda) R^{j}\left(s_{t+1}\right)-\lambda B^{j}\left(s_{t+1}\right)\right\}-\kappa \varepsilon .
\end{aligned}
$$

Buyers and sellers The number of buyers and sellers is given by:

$$
\begin{gathered}
\operatorname{Buyers}\left(s_{t}\right)=\sum_{j=i, c, v}\left(b_{t}^{j}\right)^{\prime \prime} J^{b, j}\left(s_{t}\right)+\sum_{j=i, c, v}\left(r_{t}^{j}\right)^{\prime \prime} J^{r, j}\left(s_{t}\right), \\
\operatorname{Sellers}\left(s_{t}\right)=\sum_{j=i, c, v}\left(u_{t}^{j}+\eta h_{t}^{j}\right) .
\end{gathered}
$$

The number of homes sold is given by equation (4.22). The probabilities of buying and selling are given by equations (4.23) and (4.24), respectively.

Transactions prices There are six different possible transaction prices. The first three prices arise from a match between a home seller and the three different types of natural buyers:

$$
P^{b, j}\left(s_{t}\right)=\theta \bar{P}^{b, j}\left(s_{t}\right)+(1-\theta) \bar{P}^{u} .
$$

The remaining three prices arise from a match between a home seller and the three different types of natural renters:

$$
P^{r, j}\left(s_{t}\right)=\theta \bar{P}^{r, j}\left(s_{t}\right)+(1-\theta) \bar{P}^{u} .
$$


The average price received by a seller, $P\left(s_{t}\right)$ is given by:

$$
P\left(s_{t}\right)=\frac{\sum_{j=i, v, c}\left(b_{t}^{j}\right)^{\prime \prime} J^{b, j}\left(s_{t}\right) P^{b, j}\left(s_{t}\right)+\sum_{j=i, v, c}\left(r_{t}^{j}\right)^{\prime \prime} J^{r, j}\left(s_{t}\right) P^{r, j}\left(s_{t}\right)}{\sum_{j=i, v, c}\left(b_{t}^{j}\right)^{\prime \prime} J^{b, j}\left(s_{t}\right)+\sum_{j=i, v, c}\left(r_{t}^{j}\right)^{\prime \prime} J^{r, j}\left(s_{t}\right)} .
$$

Here $J^{b, j}\left(s_{t}\right)$ is an indicator function that is equal to one if it is optimal for a type $j$ natural buyer to buy a home when the state of the economy is $s_{t}$ and zero otherwise. Similarly, $J^{r, j}\left(s_{t}\right)$ is an indicator function that is equal to one if it is optimal for a type $j$ natural renter to buy a home when the state of the economy is $s_{t}$ and zero otherwise.

\subsection{Solving the model}

In this subsection we describe a solution algorithm to compute the equilibrium of the economy along a path in which uncertainty has not been realized.

We begin by considering case 1 . In this case, absent resolution of uncertainty, all agents eventually become cured. Since $E^{c}\left(\varepsilon^{*}\right)=\varepsilon$, if the path under consideration converges then it converges to the initial steady state of the economy.

We can use the algorithm described in Section 4 to solve for the steady state associated with all possible realizations of $\varepsilon^{*}$ and for the values of the value functions along the transition to the steady state for any initial condition $s_{t}$ and realized value of $\varepsilon^{*}: \bar{H}\left(\varepsilon^{*}, s_{t}\right), \bar{U}\left(\varepsilon^{*}, s_{t}\right)$, $\bar{B}\left(\varepsilon^{*}, s_{t}\right)$, and $\bar{R}\left(\varepsilon^{*}, s_{t}\right)$. As in Section 4 we denote by $S$ the set of the values of the state variable $s_{t}$ that occur along the equilibrium path.

Our solution algorithm is as follows. First, we specify the initial conditions in the economy: $h_{0}^{j}, u_{0}^{j}, b_{0}^{j}$, and $r_{0}^{j}$ for $j=i, c, v$. We choose these conditions so that the fractions of homeowners, home sellers, natural buyers and natural renters are equal to their initial steady state values. In addition, we assume that all agents are vulnerable except for a small number, $\zeta$, of infected and cured renters: $h_{0}^{v}=h$ and $b_{0}^{v}=b, u_{0}^{v}=u, r_{0}^{i}=r_{0}^{c}=\zeta$, and $r_{0}^{v}=1-2 \zeta$.

Second, we guess values of the indicator functions that summarize the optimal decisions of natural buyers and natural renters, $J^{b, j}\left(s_{t}\right)$ and $J^{r, j}\left(s_{t}\right)$ for all $s_{t} \in S$. For example, $J^{b, j}\left(s_{t}\right)=1$ for all $j, J^{r, i}\left(s_{t}\right)=1, J^{r, c}\left(s_{t}\right)=J^{r, v}\left(s_{t}\right)=0$ for all $s_{t} \in S$.

Third, we use equations (A.1) through (A.20) in the appendix to compute the path for the fractions of different agents in the population: $h_{t}^{v}, h_{t}^{c}, h_{t}^{i}, u_{t}^{v}, u_{t}^{c}, u_{t}^{i}, b_{t}^{v}, b_{t}^{c}, b_{t}^{i}, r_{t}^{v}, r_{t}^{c}$, and 
$r_{t}^{i}$ and equations (4.23), (4.24), (5.8), and (5.9), to compute the values of $p\left(s_{t}\right)$ and $q\left(s_{t}\right)$ for $s_{t} \in S$.

Fourth, we compute the limiting value of the value function of all agents along the path in which uncertainty is not realized. The system of equations that defines these limiting values is given by equation (A.21)-(A.27) in the appendix.

Fifth, we solve backwards for all the value functions using equations (5.2) to (5.7) and (5.10) to (5.12). As in Section 4 we assume that the economy has reached its steady state at time $T=2000$. Computing $E^{j}\left[V^{j}\left(s_{t+1}\right)\right]$, defined in equation (5.1), where $V^{j}$ is a generic value function, requires solving the steady state that obtains when uncertainty is realized for each possible value of $\varepsilon^{*}$ and solving backwards from the steady state to obtain $\bar{V}\left(\varepsilon^{*}, s_{t+1}\right)$ for each possible value of $\varepsilon^{*}$. We then use the pdf of agent $j$ over $\varepsilon^{*}$ to compute the expected value of $\bar{V}\left(\varepsilon^{*}, s_{t+1}\right): \sum_{\varepsilon^{*} \in \Phi} f^{j}\left(\varepsilon^{*}\right) \bar{V}\left(\varepsilon^{*}, s_{t+1}\right)$.

Sixth, we verify that the initial guesses for the indicator functions $J^{b, j}\left(s_{t}\right)$ and $J^{r, i}\left(s_{t}\right)$ describe the optimal behavior of buyers and sellers along the proposed equilibrium path. If not, we revise the guesses until we obtain a consistent solution.

With one exception we use the same algorithm to solve for the equilibrium in case 2 . The exception is that in this case, absent resolution of uncertainty, all agents become infected. Along the path on which uncertainty is not realized the economy converges to a steady state which is equivalent to an economy in which $\varepsilon^{*}=E^{i}\left(\varepsilon^{*}\right)$. To compute this steady state we guess and verify whether it is optimal for infected natural renters and natural buyers to buy a home. In our particular numerical example, it is optimal for them to do so.

\subsection{Quantitative properties of the model}

We illustrate the properties of the model using two numerical examples corresponding to cases 1 and 2 in Section 3. The parameters governing social dynamics are the same as in Section 3. The parameters of the matching model are the same as in Section 4. Table 1 contains a summary of the parameter values that we use.

Case 1 In this case the pdf of the cured agents has the lowest entropy $\left(e^{c}<e^{i}<e^{v}\right)$. The resulting social dynamics are displayed in panel (a) of Figure 2. Figure 6 describes various features of the model along a path in which uncertainty is not realized. The key features of this path can be summarized as follows. First, average home prices rise and then fall 
as the infection waxes and wanes. Strikingly, even though agents have perfect foresight up to the resolution of long-run uncertainty, the initial rise in price is very small. Second, the average transaction price is highly correlated with the number of potential buyers. Third, the number of transactions is positively correlated with the average home price. Fourth, as prices rise there is a "sellers market" in the sense that the probability of selling is high and the probability of buying is low.

Consistent with our discussion in Section 4, movements in the number of potential buyers are the key driver of price dynamics in the model. Over time the number of potential buyers rises from 4.7 percent to a peak value of 13 percent of the population and then declines.

In the boom phase of the cycle the number of potential buyers rises for two reasons. First, in contrast to the model without social dynamics, some natural renters, those who have become infected, want to buy homes. At the peak of the infection roughly 16 percent of natural renters are infected and account for 37 percent of potential buyers (see Figure 6). Second, as more buyers enter the market, the average amount of time to purchase a house rises from 7.5 to 20 months, while the average time to sell a house drops from 7.5 months to 2.8 months. To understand these results, recall that the probabilities of buying and selling a home depends on the ratio of buyers to sellers (see equations (4.23) and (4.24)). Other things equal, the inflow of infected natural renters into the housing market increases the number of buyers, thereby lowering the probability of buying a house and raising the probability of selling a house. The latter effect reduces the stock of home sellers, thus reinforcing the fall in the probability of buying and the rise in the probability of selling a house. As the infection wanes, the number of buyers falls and the number of sellers rises, so the probabilities of buying and selling a house return to their steady state values.

To understand how changes in the number of buyers and sellers affect prices, we exploit the intuition about transition dynamics discussed in Section 4. The average purchase price is a weighted average of the price paid by four types of agents: infected natural renters and infected, cured and vulnerable natural buyers.

The price paid by each of these agents depends positively on their reservation price (see equations (5.10) and (5.11)). Each reservation price is the difference between the value to that agent of being a home owner and a home buyer (see equations (5.5) and (5.7)). When the probability of buying is low, the value functions of all potential buyers are low because it is more difficult to realize the utility gains from purchasing a home. When the probability 
of selling is high, the value functions of home sellers are high because it takes less time to sell a home. The value functions of home owners are also high because with probability $\eta$ they become home sellers.

As the infection takes hold the probability of buying falls and the probability of selling rises. As a result, the reservation prices of the different potential buyers rise, leading to a rise in purchase prices.

From Figure 6 we see that the infected natural buyers pay the highest price. These agents derive a high utility from owning a home and have a high expectation of $\varepsilon^{*}$. The next highest price is paid by cured natural buyers. These agents also derive a high utility from owning a home but they have a lower expectation of $\varepsilon^{*}$ than infected natural buyers. Vulnerable and cured natural buyers have the same expectation of $\varepsilon^{*}$ so they pay the same price. Infected natural renters pay the lowest price. On one hand these agents enjoy the house less than natural buyers. On the other hand, they have a higher expectation of $\varepsilon^{*}$ than cured and vulnerable natural buyers. For the case being considered the first effect outweighs the second effect.

The presence of infected natural renters has two effects. Taking the prices paid by other agents as given, the presence of infected natural renters reduces the average price. However, the presence of infected renters increases the number of potential buyers thereby creating a congestion effect that reduces the probability of buying a home. As discussed above, this reduction increases the transactions price paid by the other agents in the system. In our example, the second effect dominates the first effect.

Quantifying the congestion effect One way to quantify the importance of the congestion effect is to redo the experiment but not allow infected renters to purchase homes. By construction, in this experiment the probability of buying and selling a home is constant, since the number of potential buyers and sellers is unaffected by the infection. It turns out that the average sale price is hardly affected by the infection. The only reason for average prices to go up in this experiment is a rise in the reservation price of infected natural buyers. This price is the difference between the value of a being a new home owner who is infected $\left((1-\eta) H^{i}\left(s_{t+1}\right)+\eta U^{i}\left(s_{t+1}\right)\right)$ and the value of being an infected natural buyer $\left(B^{i}\left(s_{t+1}\right)\right)$. The value of becoming a home owner increases if a vulnerable agent becomes infected. But the value of being an infected natural buyer also increases because an infected agent has a high expected value of $\varepsilon^{*}$. In contrast to the situation where the congestion effect is opera- 
tive, here the probability of buying a home remains constant, so there is no countervailing effect on the infected natural buyers' value functions. The net result is a small increase in the reservation price of infected buyers.

What happens when uncertainty is resolved? The two graphs in Figure 7 show the average behavior of the price if uncertainty is realized in years five and ten, respectively. The solid line depicts the actual house price up to the period when uncertainty is realized. When uncertainty is realized there are six possible price paths that can occur, one for each of the possible realized values of $\varepsilon^{*}$. The dashed (dotted) line shows the average price path that infected (vulnerable/cured) agents expect to occur after uncertainty is realized.

On average, infected agents expect prices to rise and cured/vulnerable agents expect prices to fall. Interestingly, neither agent expects the price to converge immediately to its steady value after uncertainty is realized. The reason is that, when uncertainty is resolved, the number of buyers exceeds its steady state value. For every value of $\varepsilon^{*}$ the transition to the steady state is governed by the transition dynamics of the homogeneous expectations model. As emphasized in Section 4, when the number of buyers exceeds its steady state value the price converges to its steady state value from above.

If uncertainty is realized in year five, the number of infected natural renters is small and the number of potential buyers is close to its steady state value. As a result, there is only a modest role for transition dynamics and the expected initial price is close to its expected steady state value. If uncertainty is realized in year ten, the number of infected natural renters is large and the number of potential buyers is substantially above its steady state value. As a result, the expected initial price is substantially above its expected steady-state value.

The right-hand graph in Figure 7 helps us understand why a cured or vulnerable natural buyer is willing to buy a house even at the peak of the infection (year ten) when the price is much higher than the steady state price that these agents expect. Even if uncertainty is resolved in the following period, agents expect the fall in the price to be relatively small because the number of potential home buyers is significantly above its steady state value. Even if a home buyer becomes a home seller, the expected capital loss on the house is expected to be relatively small. As a consequence, the gains from living in the house outweigh the expected capital loss.

Infected agents expect a large capital gain when uncertainty is realized. This expected 
gain is so large that it induces not only natural buyers but also natural renters to try to purchase a home. Under normal circumstances natural renters would not buy a home. They are willing to do so because the expected gains from speculation outweigh their disposition to rent rather than buy.

Finally, Figure 7 shows that there is a discontinuous jump up or down in housing prices when uncertainty is realized. We do not observe these types of jumps in the data. The discontinuity reflects the stark nature of how information is revealed in the model. This feature can be eliminated if information about long-run fundamentals gradually percolates throughout the economy as in Duffie, Giroux and Manso (2010).

Case 2 In this case the pdf of the infected agents has the lowest entropy $\left(e^{i}<e^{c}<\right.$ $\left.e^{v}\right)$. The same economic forces discussed above are at work here. The key difference is that, absent resolution of uncertainty, the entire population becomes infected (see panel (b) of Figure 2). As a consequence, the number of infected renters rises and remains high until uncertainty is resolved. So, the number of potential buyers remains high and the congestion effect is operative for a much longer period of time. Not surprisingly, in case 2 the probability of buying (selling) is much lower (higher) for a longer period of time than in case 1. Consequently, it takes much longer in case 2 for the volume of transactions to return to its steady state level.

Finally, Figure 8 displays the price path absent resolution of uncertainty. As in case 1 , the price rises before year ten, albeit to a higher level, reflecting the larger number of potential buyers in the system. The price stays high until uncertainty is resolved.

Expected price paths We now discuss the properties of the time-zero expected time- $t$ price, $E_{0}^{j}\left(P_{t}\right)$, for $j=i, c$. This price is computed as follows:

$$
E_{0}^{j}\left(P_{t}\right)=(1-\phi)^{t} \mathcal{P}_{t}+\sum_{\tau=1}^{t} \phi(1-\phi)^{\tau-1} E_{0}^{j}\left[\mathcal{P}_{t}^{*}\left(\varepsilon^{*}, s_{\tau}\right)\right],
$$

The first term in equation (5.13) reflects the possibility that uncertainty has not yet been resolved by the end of time $t$. The probability of this event, $(1-\phi)^{t}$, is multiplied by $\mathcal{P}_{t}$, the price at time $t$ in that state of the world. The second term in equation (5.13) reflects the possibility that uncertainty is resolved at time $\tau \leq t$, an event that occurs with probability $\phi(1-\phi)^{\tau-1}$. This probability is multiplied by $E^{j}\left[\mathcal{P}_{t}^{*}\left(\varepsilon^{*}, s_{\tau}\right)\right]$, the price that agent $j$ expects 
to occur at time $t$ if uncertainty is realized at time $\tau$ and the utility of owning a home is $\varepsilon^{*}$. The time- $t$ price depends on $\tau$ because the time- $t$ state of the economy depends on when uncertainty is realized as that determines the transition path to the steady state. Finally, since agents differ in their expectations about $\varepsilon^{*}$, the expected value of $\mathcal{P}_{t}\left(\varepsilon^{*}, s_{\tau}\right)$ also differs across agents.

Panel (a) of Figure 9 depicts, for case 1, the price paths expected by different agents. Infected agents expect prices to rise rapidly until year ten and to remain high. In contrast, cured agents also expect prices to rise up to year ten, although by less than infected agents. Thereafter, cured agents expect prices to revert to their old steady state levels.

Panel (b) of Figure 9 depicts, for case 2, the price paths expected by different agents. The key property to notice is that while there are quantitative differences the patterns are remarkably similar. Cured agents expect a boom that is followed by a bust while infected agents expect a boom that is not followed by a bust. Qualitatively this is the same result we obtained with the frictionless model of Section 3. Once again, an econometrician taking repeated samples from our data would see both boom-busts and booms that are not followed by busts. The boom-bust episodes occur in economies where the cured agents happen to be correct. The booms that are not followed by busts occur in economies in which infected agents happen to be correct.

\section{Conclusion}

Boom-bust episodes are pervasive in housing markets. They occur in different countries and in different time periods. These episodes are hard to understand from the perspective of conventional models in which agents have homogeneous expectations.

In this paper we propose a model in which agents have different views about long-run fundamentals. Social interactions can generate temporary increases in the fraction of agents who hold a particular view about long-run fundamentals. The resulting dynamics can produce boom-bust cycles as well as booms that are not followed by busts. The pattern observed in a given episode depends on which set of agents happens to be correct in its views.

At the core of our matching model is the notion that booms are associated with new entrants into the market who drive up housing prices. In the model these entrants are renters who would not normally be disposed to buy a house. They do so because they expect a large capital gain. This core feature of our model is consistent with evidence that housing booms 
are accompanied by an influx of new buyers. Data from the American Housing Survey compiled by the U.S. Census Bureau shows that the fraction of homes owned by individuals 25 years old and younger increased from 18 percent in 1997 to 25 percent in 2005 . This rise surely reflects an influx of new buyers. Similarly, Ortalo-Magne and Rady (1999) document that there was an influx of first-time buyers during the 1990 housing boom in the U.K.

Our model abstracts from financial frictions. But it is clear to us that the ability of many young buyers to buy a home is influenced by downpayment requirements and credit conditions. An implication of our model is that if young buyers are infected but cannot buy a house, say because they are credit constrained, boom-bust cycles in housing prices are greatly muted. Indeed, this situation corresponds to the experiment in our model where we lock out infected natural renters from the housing market. In this case there are no congestion effects and there are no pronounced boom-bust cycles. But there is no presumption that a policy of requiring high downpayments would be welfare improving because this policy would presumably apply to both natural buyers and natural renters. More generally, policies aimed at curbing rapid price increases are not obviously welfare improving in our model because, in the end, we do not know who is right about the future: the vulnerable, the cured, or the infected. 


\section{REFERENCES}

Acemoglu, Daron, Victor Chernozhukov, and Muhamet Yildiz (2007) "Learning and Disagreement in an Uncertain World," National Bureau of Economic Research Working Paper, No. 12648.

Ambrose, Brent, Piet Eichholtz, and Thies Lindenthal (2010) "House Prices and Fundamentals: 355 years of Evidence," mimeo, Maastricht University.

Barlevy, Gadi and Jonas D.M. Fisher (2010) "Mortgage Choices and Housing Speculation," mimeo, Federal Reserve Bank of Chicago.

Bernoulli, Daniel (1766) "Essai d'une Nouvelle Analyse de la Mortalitée Causée par la Petite Vérerole," Mém. Math. Phys. Acad. Roy. Sci., Paris.

Bureau of the Census, Current Population Survey/Housing Vacancy Survey, Series H-111 Reports, Washington, DC 20233.

Chu, Yongqiang (2009) "Was it Really a Housing Bubble?" mimeo, University of Wisconsin, September.

Diba, Behzad T. and Herschel I. Grossman (1987) "On the Inception of Rational Bubbles," The Quarterly Journal of Economics 102 (3, Aug.), 697-700

Dietz, Klaus and J.A.P. Heesterbeek (2002) "Daniel Bernoulli's Epidemiological Model Revisited," Mathematical Biosciences 180, 1-21.

Duffie, Darrell and Yeneng Sun (2007) "The Existence of Independent Random Matching," Annals of Applied Probability 17, 386-419.

Duffie, Darrell, Gaston Giroux and Gustavo Manso (2010) "Information Percolation," American Economic Journal: Microeconomics 2, 100-111.

Eitrheim, Øyvind and Solveig K. Erlandsen (2004) "House Price Indices for Norway 18192003," in Historical Monetary Statistics for Norway 1819-2003, Norges Bank.

Geanakoplos, John (2010) "The Leverage Cycle," NBER Macroeconomics Annual 24, 1-66.

Gentzkow, Matthew and Jesse Shapiro (2010) "Ideological Segregation Online and Offline," National Bureau of Economics Research Working Paper No. 15916.

Glaeser, Edward L., Joseph Gyourko and Raven E. Saks (2005) "Why Have Housing Prices Gone Up?" National Bureau of Economics Research Working Paper No. 11129.

Glaeser, Edward L. and Joseph Gyourko (2006) "Housing Dynamics," National Bureau of Economics Research Working Paper No. 12787.

Harrison, J. Michael and David M. Kreps (1978) "Speculative Investor Behavior in a Stock Market with Heterogeneous Expectations," The Quarterly Journal of Economics 92 (2, May), 323-336 
Kermack, W. O. an A. G. McKendrick (1927) "A Contribution to the Mathematical Theory of Epidemics," Proceedings of the Royal Society of London. Series A, Containing Papers of a Mathematical and Physical Character 115(772) (Aug. 1), 700-721.

McPherson, Miller, Lynn Smith-Lovin, James M. Cook (2001) "Birds of a Feather: Homophily in Social Networks," Annual Review of Sociology 27, 415-444.

Mian, Atif and Amir Sufi (2010) "The Great Recession: Lessons from Microeconomic Data," American Economic Review 100 (2), 1-10.

Mullainathan, Sendhil and Andrei Shleifer (2005) "The Market for News," American Economic Review 95, 1031-1053.

Ortalo-Magne, François and Steven Rady (1999) "Boom in, Bust out: Young Households and the Housing Price Cycle," European Economic Review 43, 755-766.

Piazzesi, Monika and Martin Schneider (2009) "Momentum Traders in the Housing Market: Survey Evidence and a Search Model," American Economic Review 99 (2), 406-411.

Price, Paul C. and Eric R. Stone (2004) "Intuitive Evaluation of Likelihood Judgment Producers: Evidence for a Confidence Heuristic," Journal of Behavioral Decision Making 17 (1), 39-57.

Quigley, John and Steven Raphael (2005) "Regulation and the High Cost of Housing," American Economic Review 95, 323-328.

Saiz, Albert (2010) "The Geographic Determinants of Housing Supply," Quarterly Journal of Economics, 125, 1253-1296.

Scheinkman, José A. and Wei Xiong (2003) "Overconfidence and Speculative Bubbles," The Journal of Political Economy 111 (6, Dec.), 1183-1219.

Sniezekf, Janet A. and Lyn M. Van Swol (2001) "Trust, Confidence, and Expertise in a Judge-Advisor System," Organizational Behavior and Human Decision Processes 84 (2, Mar.), 288-307.

Stein, Jeremy (1995) "Prices and Trading Volume in the Housing Market: A Model with Down-payment Effects," The Quarterly Journal of Economics 110, 379-406.

Sunstein, Cass (2001) Republic.com Princeton University Press, Princeton N.J.

Vissing-Jørgensen, Annette (2003) "Perspectives on Behavioral Finance: Does 'Irrationality' Disappear with Wealth? Evidence from Expectations and Actions", NBER Macroeconomics Annual. 


\section{A. Appendix}

In this appendix we describe the laws of motion for the fraction of the population accounted for by the twelve types of agents in the model of Section 5. The values of $\gamma^{l j}$, which depend on the ratio of the entropies of the pdfs of agents $l$ and $j$, are defined in equation (3.3). Recall that $\gamma^{i c}=0$ in case 1 and $\gamma^{c i}=0$ in case two.

Homeowners We denote the fraction of home owners of type $j(j=c, i, v)$ in the beginning of the period, after preference shocks occur, after social interactions occur, and after purchases and sales occur by $h_{t}^{j},\left(h_{t}^{j}\right)^{\prime},\left(h_{t}^{j}\right)^{\prime \prime}$, and $h_{t+1}^{j}$, respectively. The laws of motion for these variables are given by:

$$
\begin{gathered}
\left(h_{t}^{j}\right)^{\prime}=h_{t}^{j}(1-\eta), \quad j=i, c, v, \\
\left(h_{t}^{v}\right)^{\prime \prime}=\left(h_{t}^{v}\right)^{\prime}-\gamma^{c v}\left(h_{t}^{v}\right)^{\prime} c_{t}-\gamma^{i v}\left(h_{t}^{v}\right)^{\prime} i_{t}, \\
\left(h_{t}^{c}\right)^{\prime \prime}=\left(h_{t}^{c}\right)^{\prime}+\gamma^{c v}\left(h_{t}^{v}\right)^{\prime} c_{t}-\gamma^{i c}\left(h_{t}^{c}\right)^{\prime} i_{t}+\gamma^{c i}\left(h_{t}^{i}\right)^{\prime} c_{t}, \\
\left(h_{t}^{i}\right)^{\prime \prime}=\left(h_{t}^{i}\right)^{\prime}+\gamma^{i v}\left(h_{t}^{v}\right)^{\prime} i_{t}-\gamma^{c i}\left(h_{t}^{i}\right)^{\prime} c_{t}+\gamma^{i c}\left(h_{t}^{c}\right)^{\prime} i_{t}, \\
h_{t+1}^{j}=\left(h_{t}^{j}\right)^{\prime \prime}+q\left(s_{t}\right) J^{b, j}\left(s_{t}\right)\left(b_{t}^{j}\right)^{\prime \prime}+q\left(s_{t}\right) J^{r, j}\left(s_{t}\right)\left(r_{t}^{j}\right)^{\prime \prime}, \quad j=i, c, v .
\end{gathered}
$$

Home sellers We denote the fraction of home sellers of type $j(j=c, i, v)$ in the beginning of the period, after preference shocks occur, after social interactions occur, and after purchases and sales occur by $u_{t}^{j},\left(u_{t}^{j}\right)^{\prime},\left(u_{t}^{j}\right)^{\prime \prime}$, and $u_{t+1}^{j}$, respectively. The laws of motion for these variables are given by:

$$
\begin{gathered}
\left(u_{t}^{j}\right)^{\prime}=u_{t}^{j}+\eta h_{t}^{j}, \quad j=i, c, v, \\
\left(u_{t}^{v}\right)^{\prime \prime}=\left(u_{t}^{v}\right)^{\prime}-\gamma^{c v}\left(u_{t}^{v}\right)^{\prime} c_{t}-\gamma^{i v}\left(u_{t}^{v}\right)^{\prime} i_{t}, \\
\left(u_{t}^{c}\right)^{\prime \prime}=\left(u_{t}^{c}\right)^{\prime}+\gamma^{c v}\left(u_{t}^{v}\right)^{\prime} c_{t}-\gamma^{i c}\left(u_{t}^{c}\right)^{\prime} i_{t}+\gamma^{c i}\left(u_{t}^{i}\right)^{\prime} c_{t},
\end{gathered}
$$




$$
\begin{gathered}
\left(u_{t}^{i}\right)^{\prime \prime}=\left(u_{t}^{i}\right)^{\prime}+\gamma^{i v}\left(u_{t}^{v}\right)^{\prime} i_{t}-\gamma^{c i}\left(u_{t}^{i}\right)^{\prime} c_{t}+\gamma^{i c}\left(u_{t}^{c}\right)^{\prime} i_{t} \\
u_{t+1}^{j}=\left(u_{t}^{j}\right)^{\prime \prime}-p\left(s_{t}\right)\left(u_{t}^{j}\right)^{\prime \prime}, \quad j=i, c, v .
\end{gathered}
$$

Natural buyers We denote the fraction of natural buyers of type $j(j=c, i, v)$ in the beginning of the period, after preference shocks occur, after social interactions occur, and after purchases and sales occur by $b_{t}^{j},\left(b_{t}^{j}\right)^{\prime},\left(b_{t}^{j}\right)^{\prime \prime}$, and $b_{t+1}^{j}$, respectively. The laws of motion for these variables are given by:

$$
\begin{gathered}
\left(b_{t}^{j}\right)^{\prime}=b_{t}^{j}+\lambda r_{t}^{j}, \quad j=i, c, v, \\
\left(b_{t}^{v}\right)^{\prime \prime}=\left(b_{t}^{v}\right)^{\prime}-\gamma^{c v}\left(b_{t}^{v}\right)^{\prime} c_{t}-\gamma^{i v}\left(b_{t}^{v}\right)^{\prime} i_{t}, \\
\left(b_{t}^{c}\right)^{\prime \prime}=\left(b_{t}^{c}\right)^{\prime}+\gamma^{c v}\left(b_{t}^{v}\right)^{\prime} c_{t}-\gamma^{i c}\left(b_{t}^{c}\right)^{\prime} i_{t}+\gamma^{c i}\left(b_{t}^{i}\right)^{\prime} c_{t}, \\
\left(b_{t}^{i}\right)^{\prime \prime}=\left(b_{t}^{i}\right)^{\prime}+\gamma^{i v}\left(b_{t}^{v}\right)^{\prime} i_{t}-\gamma^{c i}\left(b_{t}^{i}\right)^{\prime} c_{t}+\gamma^{i c}\left(b_{t}^{c}\right)^{\prime} i_{t}, \\
b_{t+1}^{j}=\left(b_{t}^{j}\right)^{\prime \prime}-q\left(s_{t}\right) J^{b, j}\left(s_{t}\right)\left(b_{t}^{j}\right)^{\prime \prime}, \quad j=i, c, v .
\end{gathered}
$$

Natural renters We denote the fraction of natural renters of type $j(j=c, i, v)$ in the beginning of the period, after preference shocks occur, after social interactions occur, and after purchases and sales occur by $r_{t}^{j},\left(r_{t}^{j}\right)^{\prime},\left(r_{t}^{j}\right)^{\prime \prime}$, and $r_{t+1}^{j}$, respectively. The laws of motion for these variables are given by:

$$
\begin{gathered}
\left(r_{t}^{j}\right)^{\prime}=r_{t}^{j}(1-\lambda), \quad j=i, c, v, \\
\left(r_{t}^{v}\right)^{\prime \prime}=\left(r_{t}^{v}\right)^{\prime}-\gamma^{c v}\left(r_{t}^{v}\right)^{\prime} c_{t}-\gamma^{i v}\left(r_{t}^{v}\right)^{\prime} i_{t}, \\
\left(r_{t}^{c}\right)^{\prime \prime}=\left(r_{t}^{c}\right)^{\prime}+\gamma^{c v}\left(r_{t}^{v}\right)^{\prime} c_{t}-\gamma^{i c}\left(r_{t}^{c}\right)^{\prime} i_{t}+\gamma^{c i}\left(r_{t}^{i}\right)^{\prime} c_{t}, \\
\left(r_{t}^{i}\right)^{\prime \prime}=\left(r_{t}^{i}\right)^{\prime}+\gamma^{i v}\left(r_{t}^{v}\right)^{\prime} i_{t}-\gamma^{c i}\left(r_{t}^{i}\right)^{\prime} c_{t}+\gamma^{i c}\left(r_{t}^{c}\right)^{\prime} i_{t}, \\
r_{t+1}^{j}=\left(r_{t}^{j}\right)^{\prime \prime}-q\left(s_{t}\right) J^{r, j}\left(s_{t}\right)\left(r_{t}^{j}\right)^{\prime \prime}+p\left(s_{t}\right)\left(u_{t}^{j}\right)^{\prime \prime}, \quad j=i, c, v .
\end{gathered}
$$


Limiting steady state when uncertainty is not realized We denote by $\bar{H}\left(\varepsilon^{*}\right), \bar{U}\left(\varepsilon^{*}\right)$, $\bar{B}\left(\varepsilon^{*}\right)$ and $\bar{R}\left(\varepsilon^{*}\right)$ the steady state of the value functions of different agents in the economy when uncertainty is realized and the realized utility of owning a home is $\varepsilon^{*}$. These values are computed by solving the system of equations (4.3), (4.4), (4.5), (4.6), (4.9), (4.8), and (4.21), setting $B=B^{\text {buy }}$ and $R=R^{\text {rent }}$ and replacing $\varepsilon$ in equation (4.3) with the different possible values of $\varepsilon^{*}$.

The limiting value functions of different agents along a path in which uncertainty is not resolved can be obtained by solving the following system of equations for $H^{j}, U^{j}, B^{j}, R^{j}$ for $j=i, v, c$ and $\bar{P}^{b, c}$ and $P$ :

$$
\begin{aligned}
& \left.H^{j}=\varepsilon+\beta\left\{(1-\phi)\left[(1-\eta) H^{j}+\eta U^{j}\right]\right)+\phi E^{j}\left[(1-\eta) \bar{H}\left(\varepsilon^{*}\right)+\eta \bar{U}\left(\varepsilon^{*}\right)\right]\right\}, \\
& U^{j}=p\left\{P+\beta\left[(1-\phi) R^{j}+\phi E^{j} \bar{R}\left(\varepsilon^{*}\right)\right]\right\}+(1-p) \beta\left[(1-\phi) U^{j}+\phi E^{j} \bar{U}\left(\varepsilon^{*}\right)\right], \\
& B^{j}=q\left(\varepsilon^{b}-w-P\right)+q \beta(1-\phi)\left[(1-\eta) H^{j}+\eta U^{j}\right] \\
& +q \beta \phi E^{j}\left[(1-\eta) \bar{H}\left(\varepsilon^{*}\right)+\eta \bar{U}\left(\varepsilon^{*}\right)\right] \\
& +(1-q)\left[\varepsilon^{b}-w+(1-\phi) \beta B^{j}+\phi \beta E^{j} \bar{B}\left(\varepsilon^{*}\right)\right], \\
& R^{j}=\varepsilon^{r}-\omega+\beta(1-\phi)\left[(1-\lambda) R^{j}+\lambda B^{j}\right]+\beta \phi E^{j}\left[(1-\lambda) \bar{R}\left(\varepsilon^{*}\right)+\lambda \bar{B}\left(\varepsilon^{*}\right)\right] .
\end{aligned}
$$

Recall that in the limit all agents are cured so the price of a home is determined by the reservation price of the natural buyer:

$$
\begin{gathered}
\bar{P}^{b, c}=\beta(1-\phi)\left[(1-\eta) H^{c}+\eta U^{c}-B^{c}\right]+\beta \phi E^{c}\left[(1-\eta) \bar{H}\left(\varepsilon^{*}\right)+\eta \bar{U}\left(\varepsilon^{*}\right)-\bar{B}\left(\varepsilon^{*}\right)\right], \\
P=\theta \bar{P}^{b, c}+(1-\theta) \bar{P}^{u} .
\end{gathered}
$$

The probability of buying and selling are given by:

$$
p=q=\mu .
$$


FIGURE 1: Real Home Prices in 18 OeCD Countries
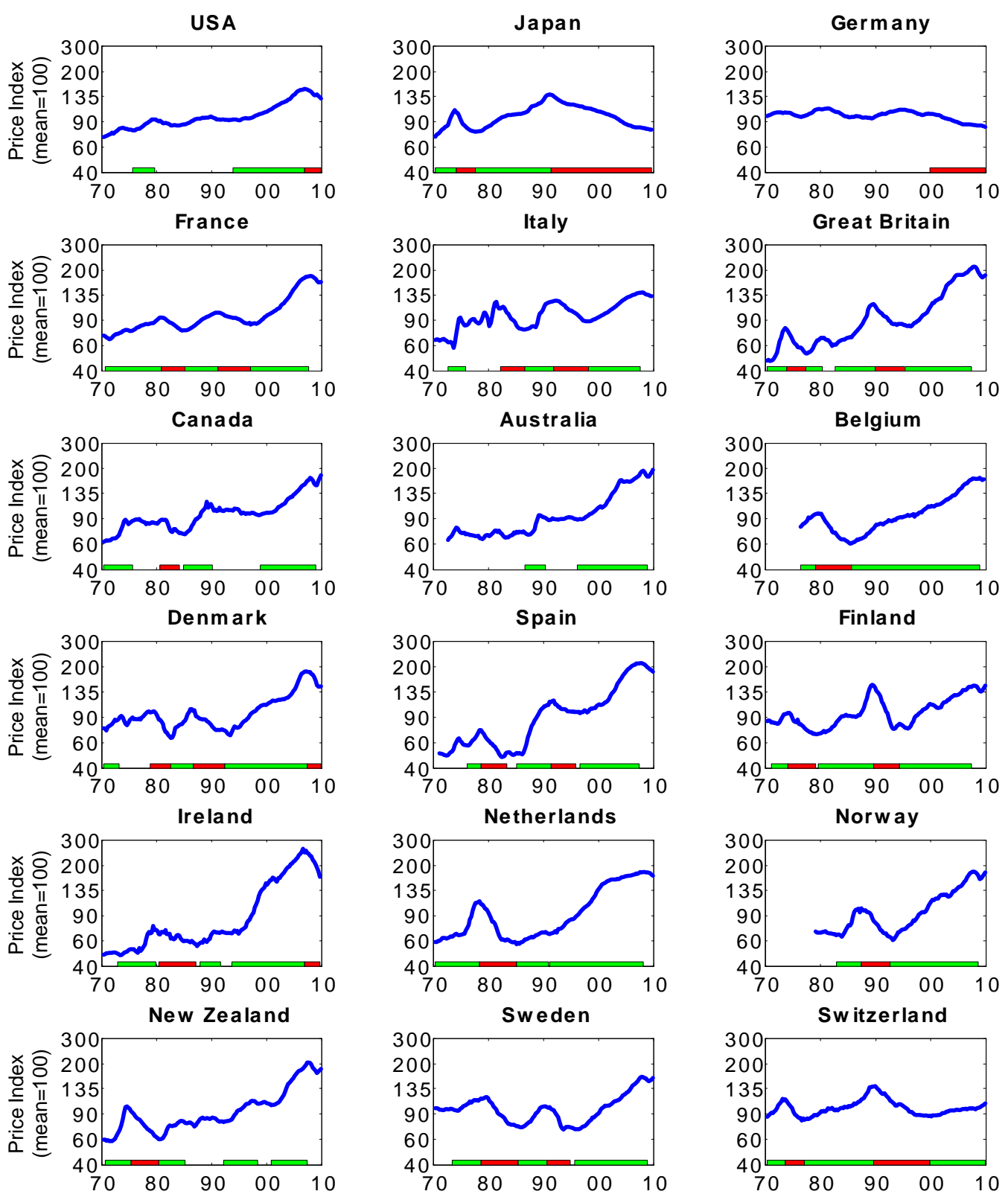

Note: Source, OECD. Each series is a nominal housing price index divided by the local CPI. The resulting series is normalized to have a mean of 100 over the full sample. Data are shown on a logarithmic scale. Green bars indicate real estate booms, red bars indicate real estate busts, as defined in the text. 
(a) CASE 1
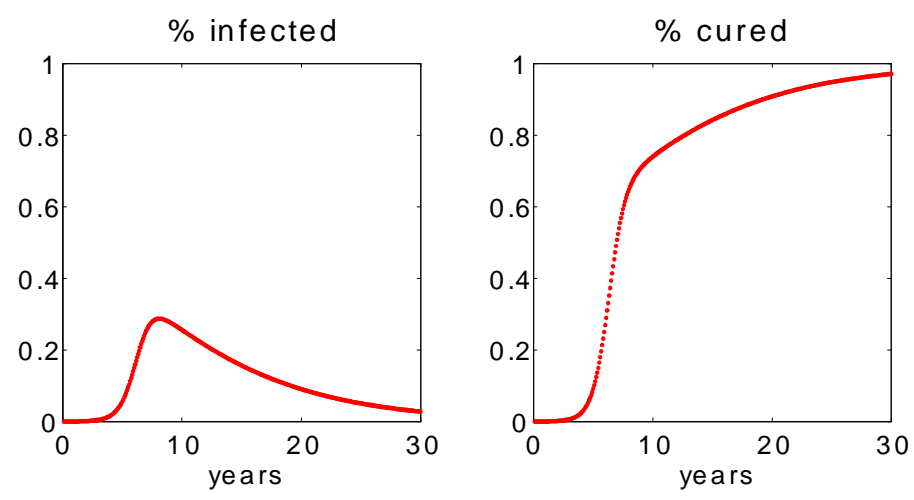

(b) CASE 2

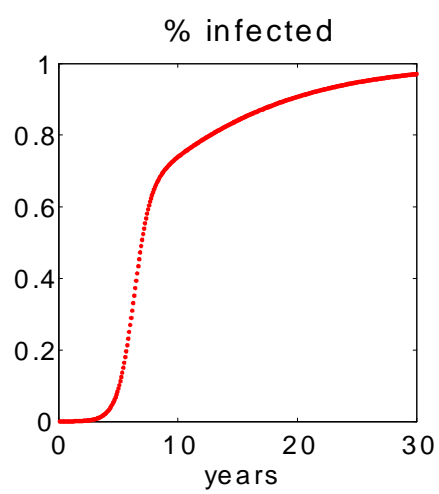

$\%$ cured

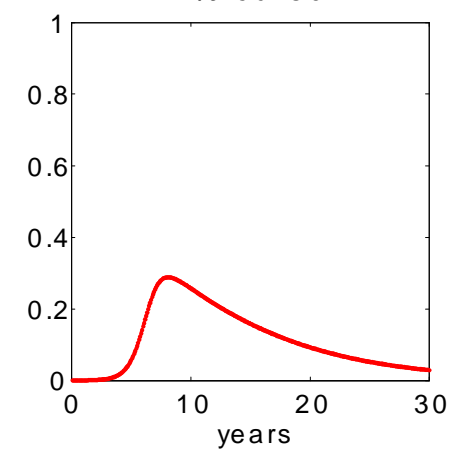

$\%$ vulnerable
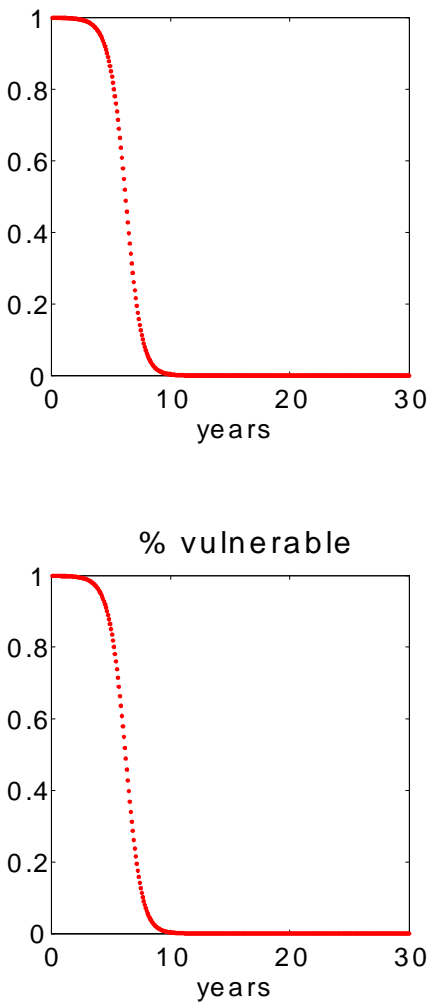

Note: The graphs show the evolution of the populations of each type of agent due to social dynamics. In Case 1, the priors of cured agents have the lowest entropy, and the priors of the vulnerable agents have the highest entropy. In Case 2, the priors of infected agents have the lowest entropy, and the priors of the vulnerable agents have the highest entropy. 

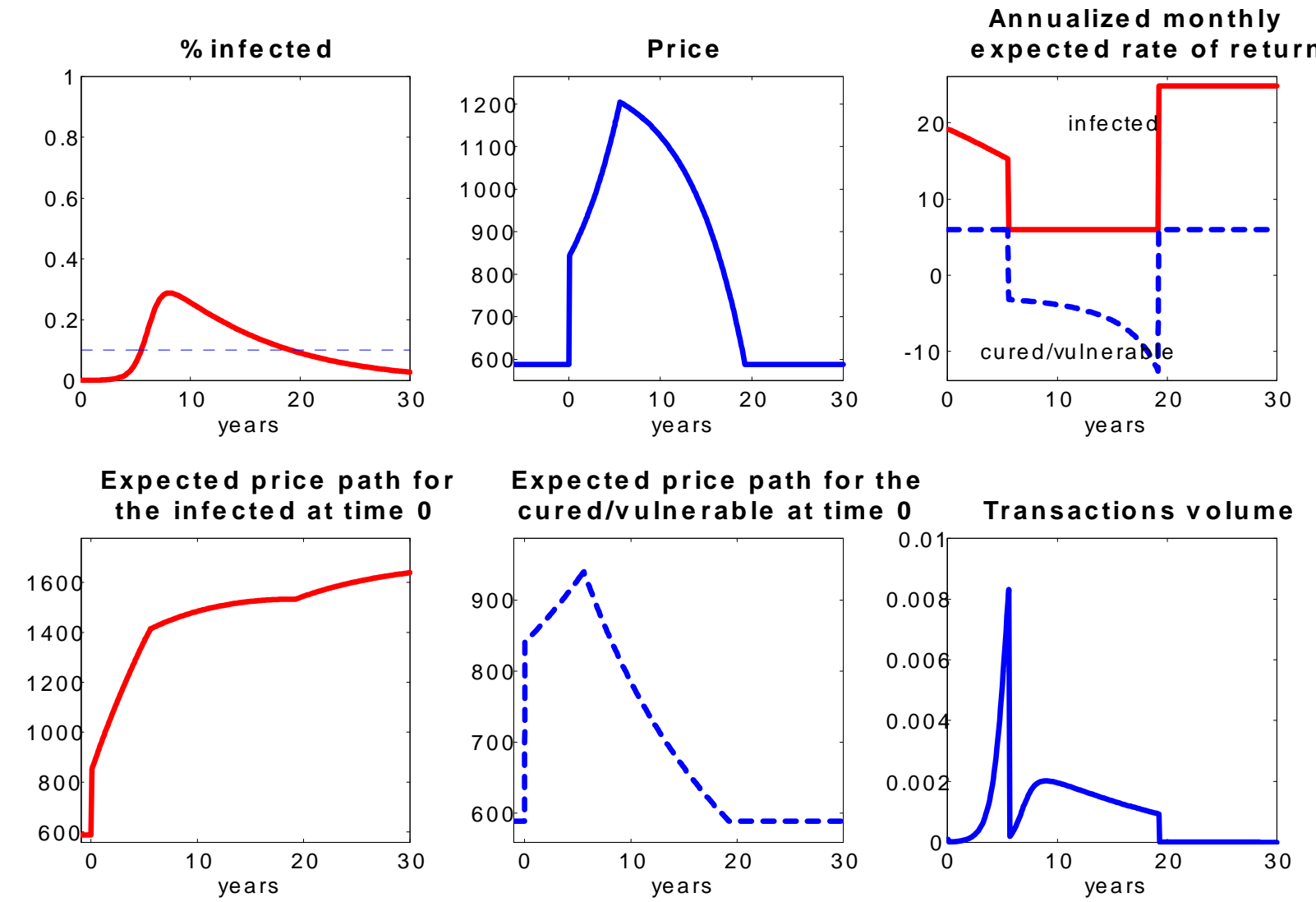

Note: The graphs show a variety of paths for the frictionless model with social dynamics in Case 1, in which the priors of cured agents have the lowest entropy, and the priors of the vulnerable agents have the highest entropy. The number of infected agents, the price of a house, the monthly expected rate of return, and transactions volume are all computed under the assumption that uncertainty is not realized. The expected price paths are the expected values of the house price at each date, as of time 0 , given the priors of the different agents. 

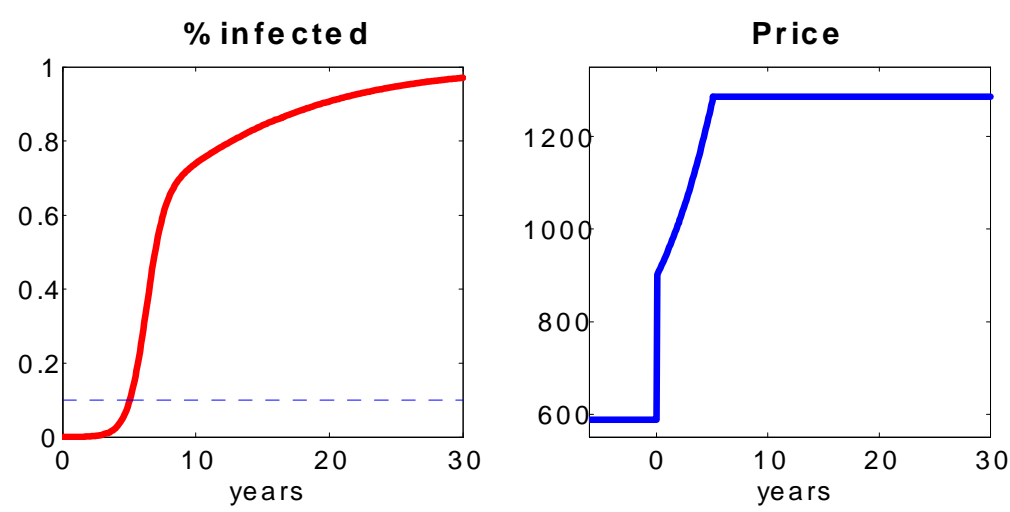

Annualized monthly expected rate of return

Expected price path for the infected at time 0
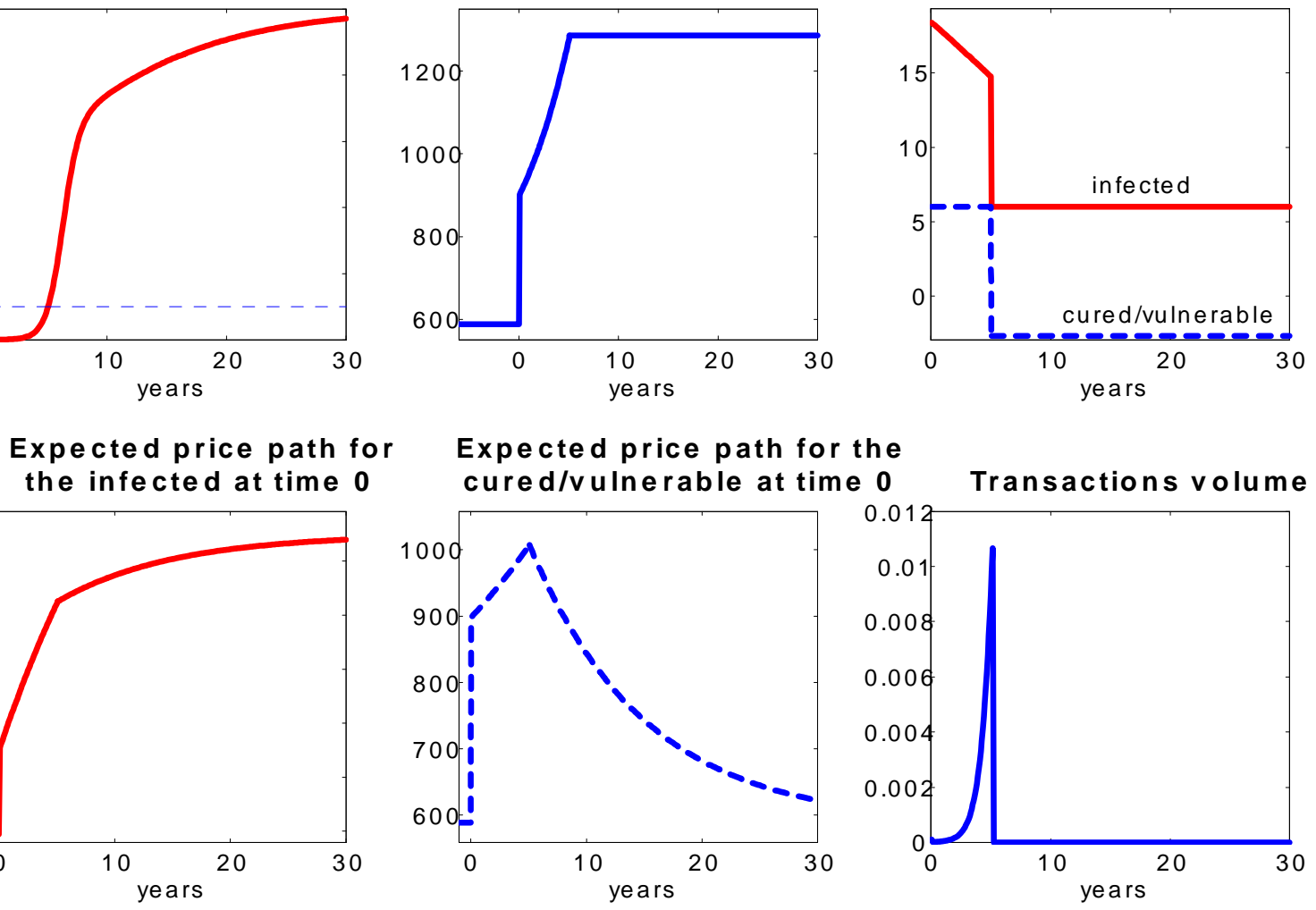

Note: The graphs show a variety of paths for the frictionless model with social dynamics in Case 2, in which the priors of infected agents have the lowest entropy, and the priors of the vulnerable agents have the highest entropy. The number of infected agents, the price of a house, the monthly expected rate of return, and transactions volume are all computed under the assumption that uncertainty is not realized. The expected price paths are the expected values of the house price at each date, as of time 0 , given the priors of the different agents. 
FigURE 5: Transitional Dynamics in a Matching Model

(a) Prices, Buyers, Sellers and Transaction Probabilities
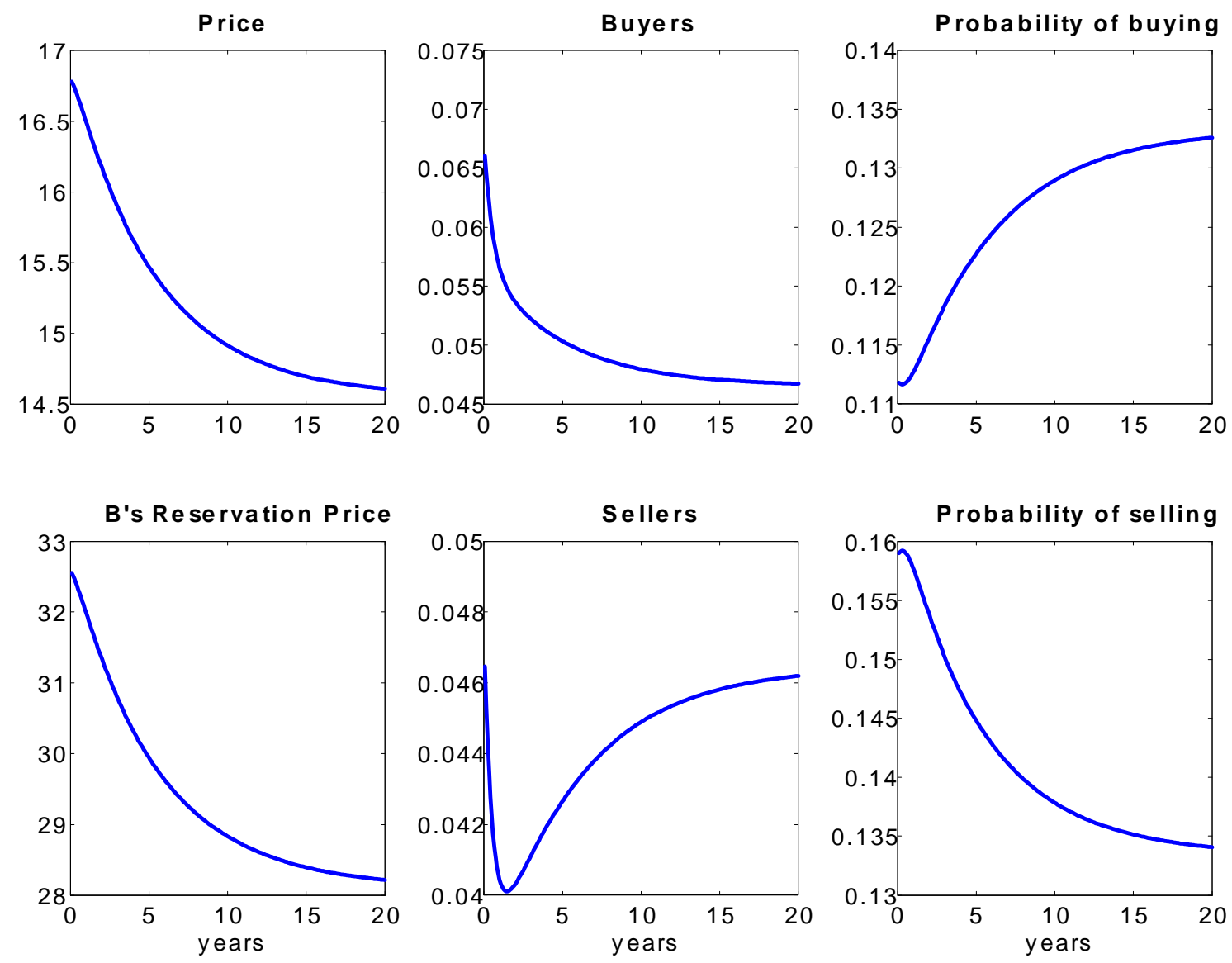

Note: The figures illustrate the transition dynamics associated with the matching model, when there is an initial increase in the number of natural home buyers. Buyers indicates the number of agents who try to buy a home, while sellers indicates the number of agents who try to sell a home. Price is the average price at which homes are sold. B's reservation price is the reservation price of a natural home buyer. Figure 5 continues on the next page. 
FigURE 5: Transitional Dynamics in a Matching Model

(b) Utility Levels of the Different Agents
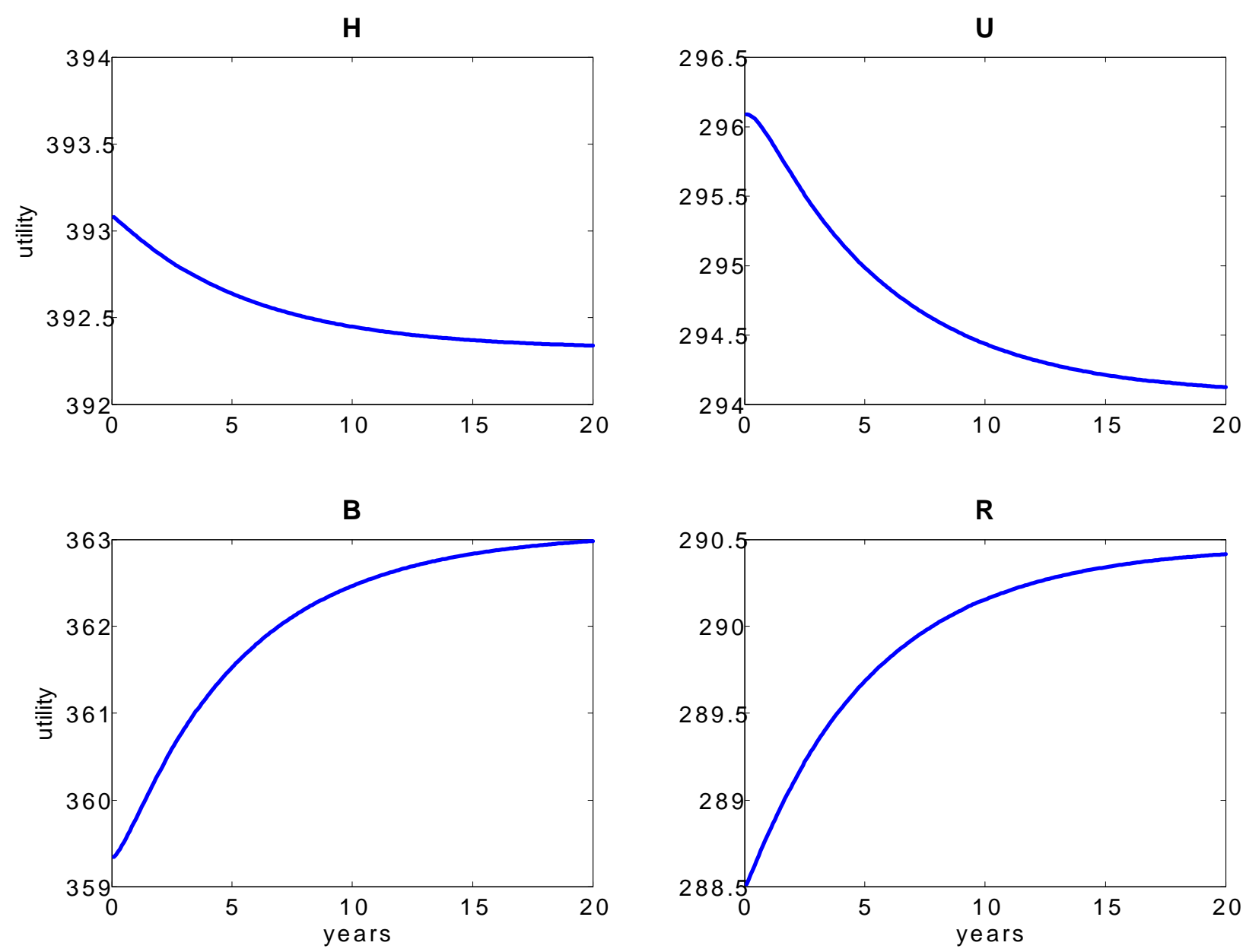

Note: The figures illustrate the transition dynamics associated with the matching model, when there is an initial increase in the number of natural home buyers. The utility levels of the four types of agents are indicated. Figure 5 continues on the next page. 
Figure 5: Transitional Dynamics in a Matching Model

(c) Agent Populations, Transactions Volume and Transaction Probabilities
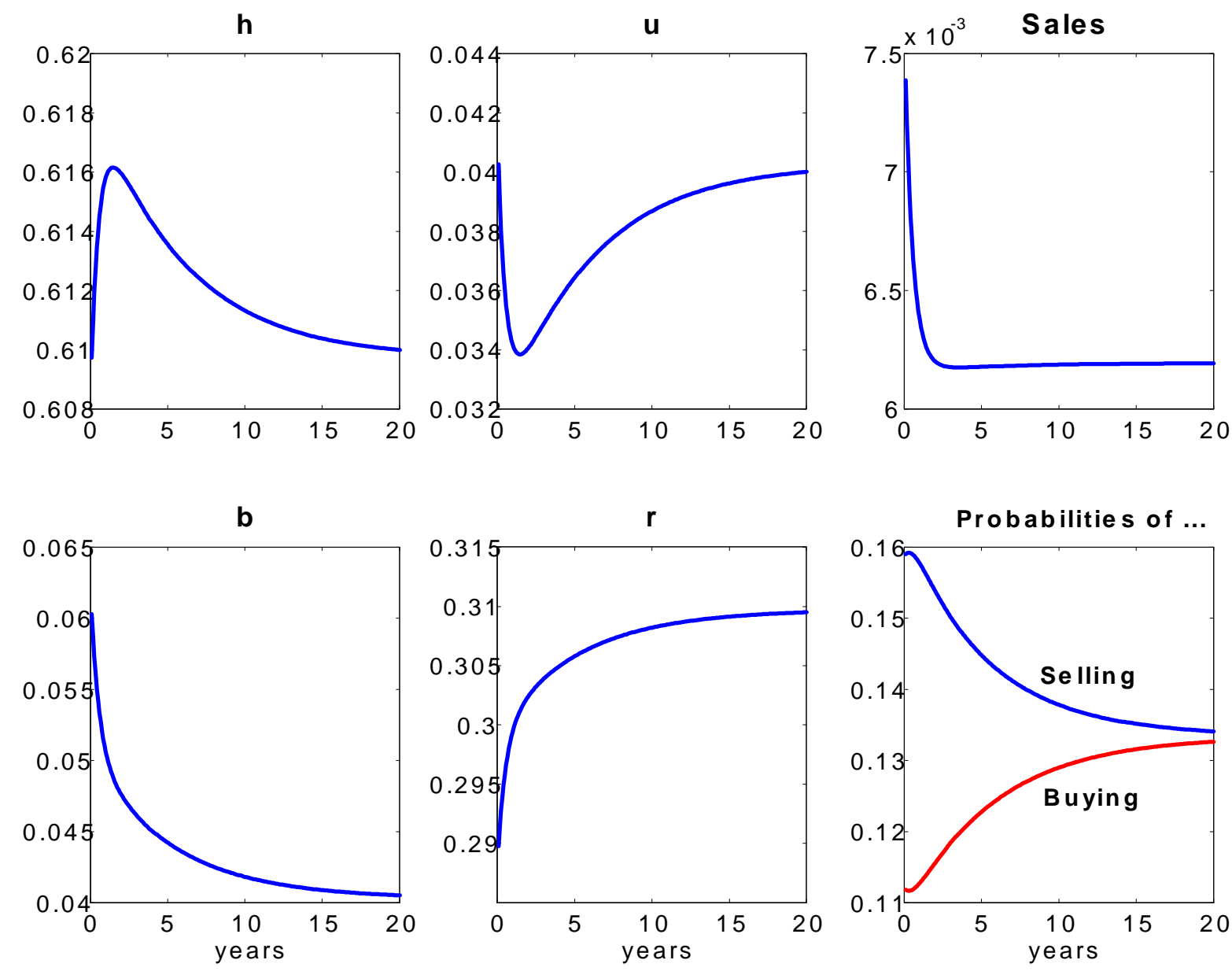

Note: The figures illustrate the transition dynamics associated with the matching model, when there is an initial increase in the number of natural home buyers. The four plots on the left show the number of agents of each type across the transition path. Sales indicates the number of transactions. 

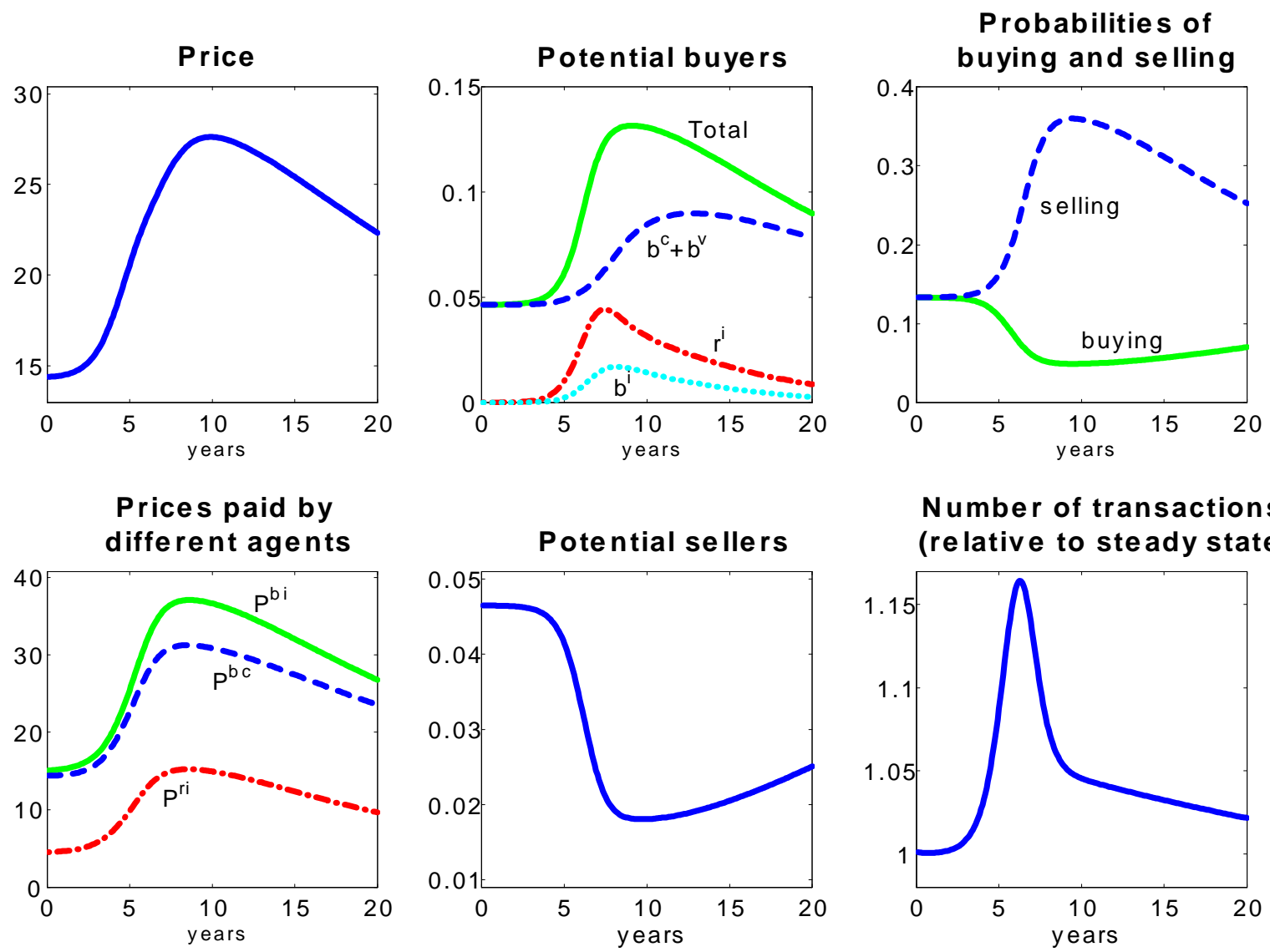

Number of transactions (relative to steady state)

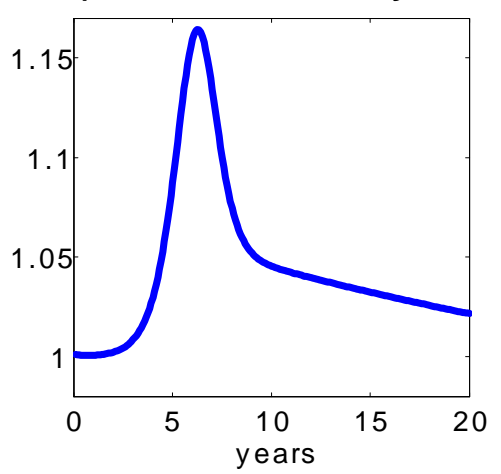

Note: The figures illustrate equilibrium paths when there is no resolution of uncertainty for the matching model with social dynamics in Case 1, in which the priors of cured agents have the lowest entropy, and the priors of the vulnerable agents have the highest entropy. The number of potential buyers is calculated after preferences shocks have been realized. Here, $b^{c}, b^{v}$ and $b^{i}$ represent the populations of cured, vulnerable and infected natural buyers, while $r^{i}$ is the population of infected natural renters. When an infected natural buyer matches with a seller he pays $P^{b i}$ for a house, a cured or infected natural buyer pays $P^{b c}$, and an infected natural renter pays $P^{r i}$. 

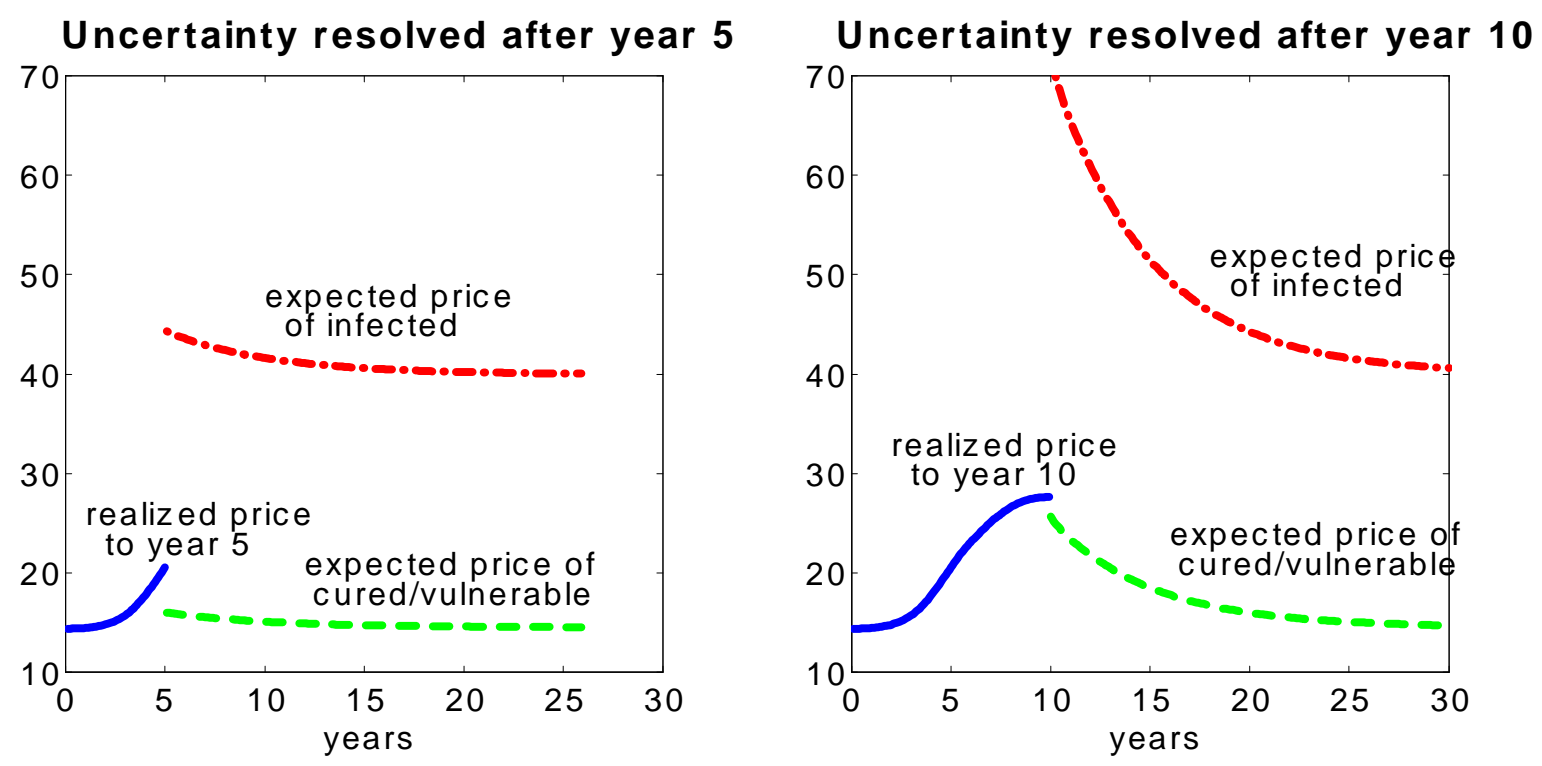

Note: The figures illustrate the equilibrium price (blue line) if uncertainty is not realized until the end of years 5 and 10, respectively. The red lines indicate the prices infected agents would expect to observe after years 5 and 10, if uncertaimty were resolved at those dates. The green lines indicate the prices the cured and vulernable agents would expect to observe after years 5 and 10, if uncertaimty were resolved at those dates. The graphs are drawn for the matching model with social dynamics in Case 1, in which the priors of cured agents have the lowest entropy, and the priors of the vulnerable agents have the highest entropy. 

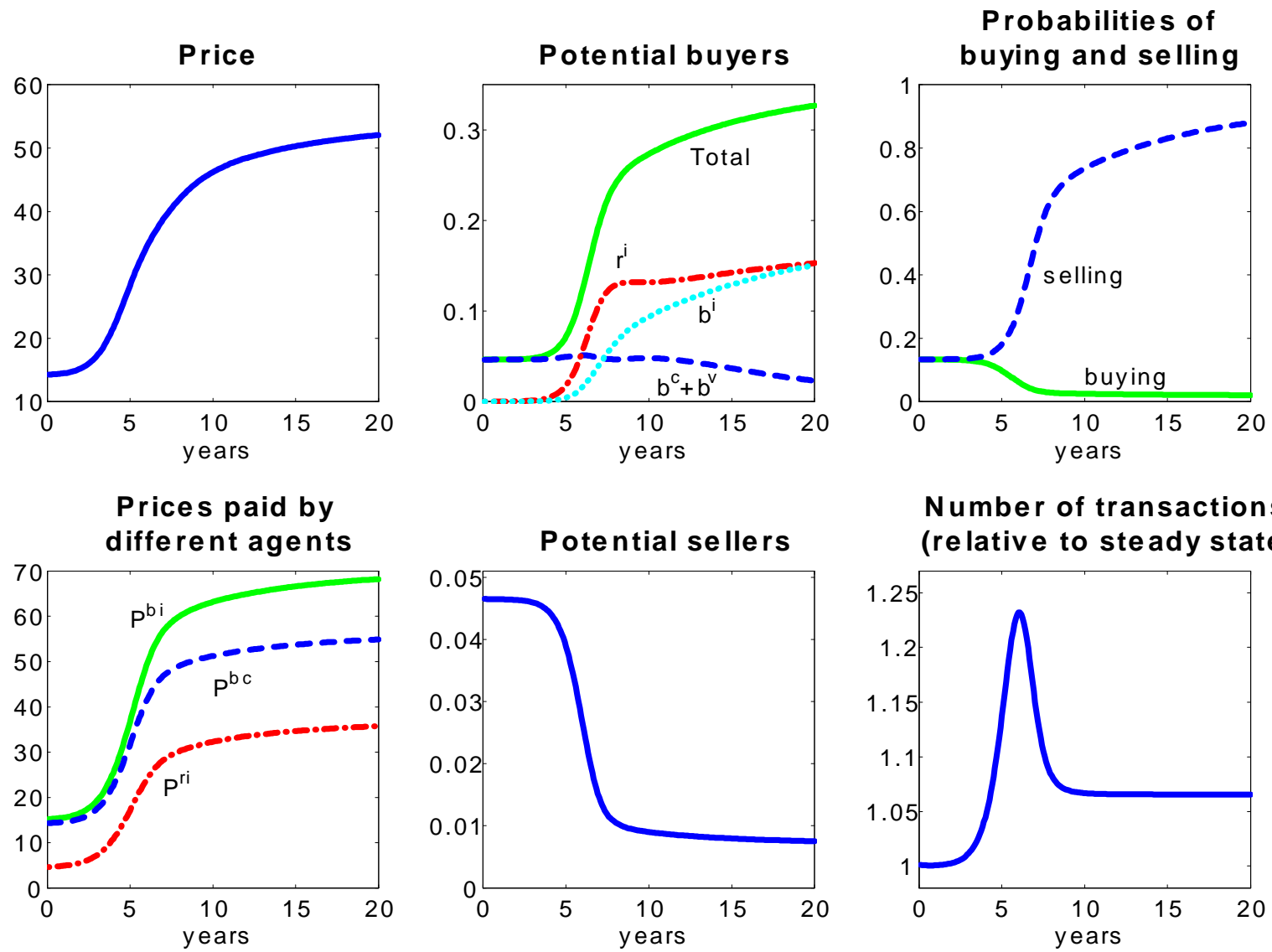

Number of transactions (relative to steady state)

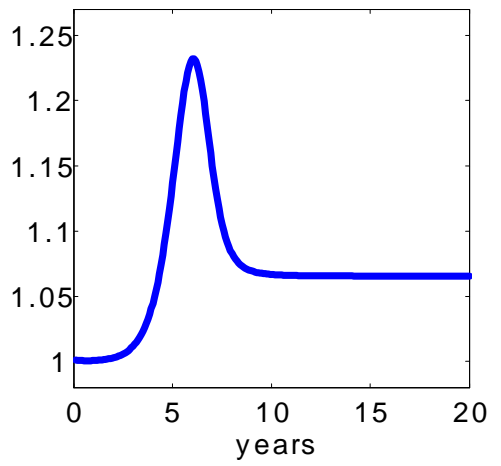

Note: The figures illustrate equilibrium paths when there is no resolution of uncertainty for the matching model with social dynamics in Case 2, in which the priors of infected agents have the lowest entropy, and the priors of the vulnerable agents have the highest entropy. The number of potential buyers is calculated after preferences shocks have been realized. Here, $b^{c}, b^{v}$ and $b^{i}$ represent the populations of cured, vulnerable and infected natural buyers, while $r^{i}$ is the population of infected natural renters. When an infected natural buyer matches with a seller he pays $P^{b i}$ for a house, a cured or infected natural buyer pays $P^{b c}$, and an infected natural renter pays $P^{r i}$. 
(a) CASE 1
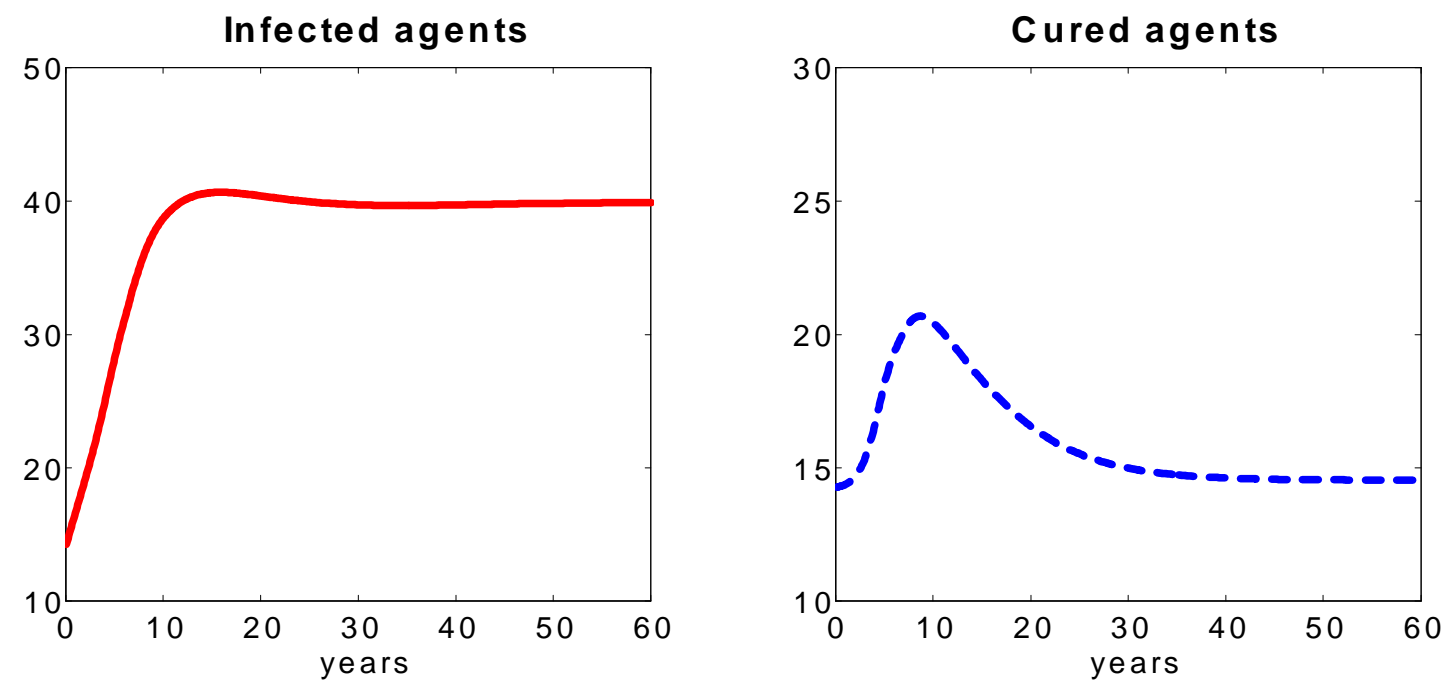

(b) CASE 2
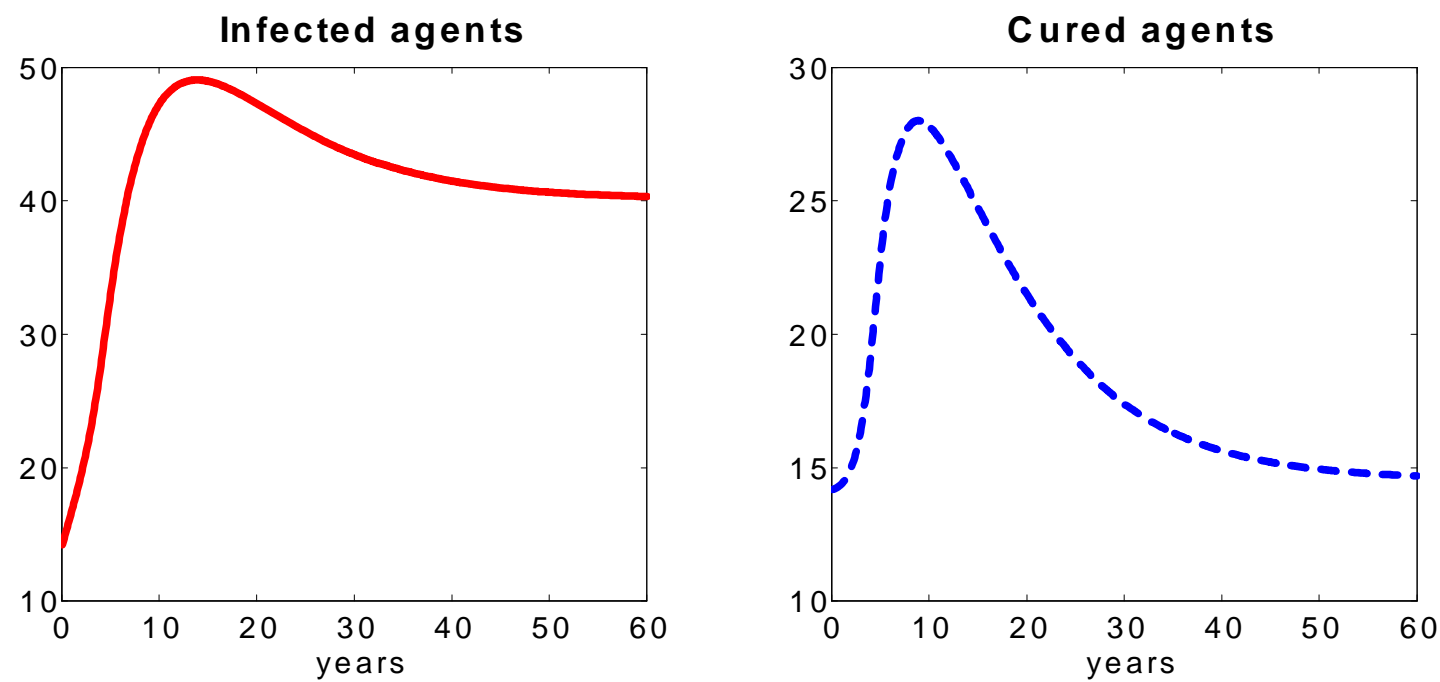

Note: The graphs show the price paths expected by different types of agents at time 0 . In Case 1, the priors of cured agents have the lowest entropy, and the priors of the vulnerable agents have the highest entropy. In Case 2, the priors of infected agents have the lowest entropy, and the priors of the vulnerable agents have the highest entropy. 
Table 1: Parameter Values, Matching Model

\begin{tabular}{lll}
\hline \hline Parameter & Value & Description \\
\hline$k$ & 0.65 & Fraction of homeowners in population \\
$\beta$ & 0.995 & Discount factor \\
$\varepsilon$ & 2.94 & Utility of owning a home \\
$\varepsilon^{b}$ & 1.00 & Utility of renting, natural buyer \\
$\varepsilon^{r}$ & 1.00 & Utility of renting, natural renter \\
$w$ & 1.00 & Rental rate \\
$\phi$ & 0.0083 & Monthly probability that uncertainty is realized \\
$\alpha$ & 0.50 & Parameter of matching function \\
$\mu$ & 0.13 & Parameter of matching function \\
$\lambda$ & 0.02 & Preference shock, natural renters \\
$\eta$ & 0.0103 & Preference shock, home owners \\
$\bar{P}^{u}$ & 1.00 & Reservation price, home sellers \\
$\kappa$ & 42 & Fixed cost of buying, natural renters \\
$\theta$ & 0.50 & Bargaining power of home buyer \\
\hline
\end{tabular}

